\title{
Conservación
}

\section{Patrones de distribución y zonas prioritarias para la conservación de la avifauna de la costa del Pacífico de Guerrero, México}

\author{
Distributional patterns and priority conservation areas for the avifauna in the Pacific coastal plain of \\ Guerrero, Mexico \\ Nefris E. Jacinto-Flores ${ }^{a}$, Luis A. Sánchez-González ${ }^{\mathrm{b}}$ y R. Carlos Almazán-Núñez ${ }^{\mathrm{c}, *}$

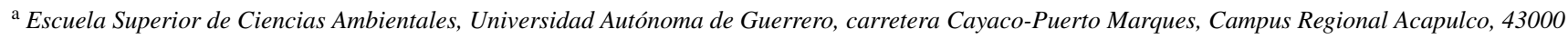 \\ Acapulco, Guerrero, México \\ ${ }^{\mathrm{b}}$ Museo de Zoología «Alfonso L. Herrera», Departamento de Biología Evolutiva, Facultad de Ciencias, Universidad Nacional Autónoma de México, Apartado \\ postal 70-399, 04510 Ciudad de México, México \\ ${ }^{\mathrm{c}}$ Laboratorio Integral de Fauna Silvestre, Facultad de Ciencias Químico-Biológicas, Universidad Autónoma de Guerrero, Av. Lázaro Cárdenas s/n, Ciudad \\ Universitaria, 39000 Chilpancingo, Guerrero, México
}

Recibido el 18 de noviembre de 2016; aceptado el 23 de mayo de 2017

Disponible en Internet el 28 de noviembre de 2017

\begin{abstract}
Resumen
Se modelaron las condiciones de idoneidad ambiental asociada a la presencia de 370 especies de aves en la costa del Pacífico del estado de Guerrero. Con estos modelos se obtuvieron los patrones de riqueza, de endemismo y de acumulación de especies de las aves en riesgo de la región. Se utilizó un análisis de complementariedad para la avifauna residente y acuática y se empleó un análisis de parsimonia de endemismos con el fin de identificar sitios prioritarios para la conservación. El conocimiento de la avifauna de la región de estudio no es completo según el estimador de riqueza (ICE: $77 \%$ del total). Los patrones de distribución de la riqueza y el endemismo de las aves de la región estuvieron más concentrados hacia el centro oeste, en las áreas montañosas de la zona de estudio, excepto en la porción más occidental que también presentó un amplio número de especies en las partes bajas coincidiendo con algunos sistemas lacustres. El análisis de complementariedad para las aves terrestres identificó 6 celdas, el mismo número de celdas para las aves acuáticas, y que en ambos casos albergan en conjunto el 100\% de las especies modeladas. El análisis de parsimonia encontró 2 áreas de endemismo y algunas celdas incluidas en estas fueron también identificadas con el método de complementariedad, por lo que el nivel de priorización es mayor. Las áreas de conservación identificadas indican una conectividad del centro al occidente del área de estudio, región que coincide con lo encontrado por otros estudios que utilizan especies importantes para la conservación. (C) 2017 Universidad Nacional Autónoma de México, Instituto de Biología. Este es un artículo Open Access bajo la licencia CC BY-NC-ND (http://creativecommons.org/licenses/by-nc-nd/4.0/).
\end{abstract}

Palabras clave: Distribución potencial; Riqueza; Endemismo; Complementariedad; Análisis de parsimonia de endemismos

\begin{abstract}
We modeled the environmental suitability conditions associated to the presence of 370 bird species along the Pacific coast of Guerrero state. Based on distribution models, regional patterns of richness, endemism and the accumulation at-risk species of birds were obtained. We used a complementarity analysis both for resident and aquatic birds and parsimony analysis of endemicity was performed in order to identify priority sites for conservation. Knowledge of the birds of the study area is incomplete according to the richness estimator (ICE: 77\% of the total). The distributional patterns of bird species richness and endemism were more concentrated in the central-western area of the study region in the mountainous areas, except in the westernmost portion that also presented a large number of species in the lower areas coinciding with some lacustrine systems. The
\end{abstract}

\footnotetext{
* Autor para correspondencia.

Correo electrónico: rcarlos.almazan@ gmail.com (R.C. Almazán-Núñez).

La revisión por pares es responsabilidad de la Universidad Nacional Autónoma de México.
} 
complementarity analysis for terrestrial birds identified 6 cells, the same number of cells for aquatic birds, and in both cases together harbored $100 \%$ of all species that were modeled. The parsimony analysis showed 2 areas of endemism, and some cells included in these areas were also identified in the complementarity analysis; hence, these areas should receive a higher level of prioritization. The conservation areas obtained in this study suggest a connectivity from the center to the west of the study area, region that coincides with findings in other studies using species importance for conservation.

(C) 2017 Universidad Nacional Autónoma de México, Instituto de Biología. This is an open access article under the CC BY-NC-ND license (http://creativecommons.org/licenses/by-nc-nd/4.0/).

Keywords: Potential distribution; Richness; Endemism; Complementarity; Parsimony analysis of endemicity

\section{Introducción}

Durante las últimas 2 décadas, y ante la acelerada pérdida de los ambientes naturales por los constantes cambios en el uso del suelo y la fragmentación de los hábitats, los estudios sobre los patrones de distribución de las especies se han incrementado, constituyéndose en una tarea prioritaria para el desarrollo de estrategias de conservación adecuadas (Koleff y Urquiza-Haas, 2011; Koleff et al., 2008; Ortega-Huerta y Peterson, 2004). Esto es particularmente importante en regiones con alta diversidad biológica, donde las tendencias de disminución de los hábitats originales se han incrementado y las áreas naturales protegidas (ANP) están poco representadas. El estado de Guerrero es un ejemplo de lo anterior, ya que es considerado como una de las 4 entidades de mayor diversidad biológica en México (Botello, Sánchez-Cordero y Ortega-Huerta, 2015), presenta una pérdida de más del 30\% de sus hábitats naturales (Semarnat, 2009) y sus bosques templados y selvas tropicales han sido transformadas en más del 50 y el 85\%, respectivamente (Semarnat, 2014). Es además la entidad con menor superficie en cuanto a ANP se refiere en México, con menos del $0.1 \%$ de su superficie dedicada a la conservación (Bezaury-Creel, Torres, Ochoa-Ochoa y Castro-Campos, 2012; Koleff y Moreno, 2006).

Varias especies o poblaciones locales prioritarias para la conservación no están representadas dentro de las ANP en el estado de Guerrero (e. g., aves: Lophornis brachrylophus, Ara militaris, Amazona oratrix; mamíferos: Panthera onca, Spilogale pygmaea), aun cuando sus hábitats se han reducido de forma significativa (Almazán-Núñez, Almazán-Juárez y Ruiz-Gutiérrez, 2011; Botello et al., 2015; Cuervo-Robayo y Monroy-Vilchis, 2012; Ramírez-Bastida, Navarro-Sigüenza y Peterson, 2008; Sierra-Morales, Almazán-Núñez, BeltránSánchez, Ríos-Muñoz y Arizmendi, 2016). Lo anterior pone de manifiesto la importancia de continuar con el diseño y la identificación de nuevas áreas para la conservación en el estado, que complementen las ya existentes y que aseguren la permanencia de su diversidad biológica a largo plazo. Aunque las ANP no son los únicos instrumentos de conservación, el establecimiento de estas áreas representa una estrategia efectiva para la protección de la biodiversidad (Ortega-Huerta y Peterson, 2004). En este sentido, durante las últimas 2 décadas en México, los esfuerzos de conservación se han enfocado en implementar métodos de análisis relativamente rápidos y eficaces para definir, complementar y evaluar objetivamente estas áreas (Botello et al., 2015; Margules y Sarkar, 2009; Navarro-Sigüenza et al., 2011; Ortega-Huerta y Peterson, 2004;
Peterson, Egbert, Sánchez-Cordero y Price, 2000; Peterson, Sánchez-Cordero, Martínez-Meyer y Navarro-Sigüenza, 2006; Suárez-Mota, Villaseñor y López-Mata, 2015). Para tal fin, algunas herramientas informáticas (e.g., el modelado de nichos ecológicos) ofrecen mayor precisión para el estudio de la distribución espacial de las especies (Guisan y Zimmermann, 2000; Guisan et al., 2006). El modelado de nicho ecológico o modelado de la distribución potencial de las especies (MDE; sensu Soberón, Osorio-Olvera y Peterson, 2017) representa un enfoque teórico-metodológico ampliamente usado en el análisis de patrones de distribución (Escalante, Sánchez-Cordero, Morrone y Linaje, 2007; Ochoa-Ochoa y Flores-Villela, 2002; Rojas-Soto, Alcántara-Ayala y Navarro-Sigüenza, 2003; Rovito, Arroyo y Pliscoff, 2004) y en el establecimiento de prioridades de conservación (Almazán-Núñez, López-de Aquino, Ríos-Muñoz y Navarro-Sigüenza, 2013; Botello et al., 2015; Illoldi-Rangel et al., 2008; Ochoa-Ochoa y FloresVillela, 2002; Suárez-Mota et al., 2015; Urbina-Cardona y Flores-Villela, 2010), especialmente con grupos bióticos que pueden ser usados como subrogados de la biodiversidad y que son clave en las decisiones de conservación, como las aves (Loiselle et al., 2003; Peterson y Navarro-Sigüenza, 2016).

Adicionalmente, algunos métodos iterativos para la identificación de áreas relevantes para la conservación de la biodiversidad utilizan principios básicos en biología de la conservación, como la complementariedad (Gil y Moreno, 2007; Rodrigues y Brooks, 2007; Rodrigues, Gregory y Gaston, 2000; Sánchez-Cordero, Peterson y Escalante-Pliego, 2001; VaneWright, Humphries y Williams, 1991) y la irremplazabilidad (Méndez-Larios et al., 2005; Pressey, Johnson y Wilson, 1994) de las áreas. Con la complementariedad, se prioriza la conservación de la máxima diversidad biológica en un número mínimo de áreas (Vane-Wright et al., 1991); por su parte, la irremplazabilidad es un concepto ligado a las especies de distribución restringida que se presentan en un área (Pressey et al., 1994). Cuando muchas de estas especies se encuentran en una región, su valor de irremplazabilidad es elevado, por lo que las prioridades de conservación aumentan (Vane-Wright, 1996). Este procedimiento es análogo a la detección de áreas de endemismo mediante el análisis de parsimonia de endemismos (PAE, por sus siglas en inglés), las cuales son definidas por patrones de distribución congruentes entre 2 o más especies (Escalante y Morrone, 2003; Morrone, 1994). Las áreas detectadas están definidas por especies que pueden considerarse exclusivas e irremplazables $\mathrm{y}$, por tanto, altamente importantes para la 
conservación (Escalante, 2009; Méndez-Larios et al., 2005; Rovito et al., 2004).

Debido a que la complementariedad y la irremplazabilidad utilizan conceptos distintos (riqueza total y especies de distribución restringida respectivamente), es importante la aplicación de ambos con el fin de desarrollar una estrategia integral de conservación (Suárez-Mota et al., 2015; Zamora-Manzur, Parra y Jaque, 2011). La aplicación de estos métodos involucra la obtención de datos primarios (presencia de las especies), los cuales corresponden a sitios puntuales de registro. Sin embargo, se ha demostrado que el uso de datos puntuales únicamente puede conducir a resultados imprecisos dada la dispersión de los datos en una gradilla (e. g., Rojas-Soto et al., 2003; SánchezGonzález, Morrone y Navarro-Sigüenza, 2008). En este sentido, los MDE proporcionan resultados con mayor certidumbre, debido a que la distribución de las especies se describe a través de áreas y no solo mediante puntos de registro. Los MDE han facilitado la identificación de áreas complementarias (Loiselle et al., 2003) y áreas de endemismo (Almazán-Núñez et al., 2013; Escalante et al., 2007; Rojas-Soto et al., 2003; SánchezGonzález et al., 2008), posibilitando el desarrollo de nuevas perspectivas para la planeación sistemática de la conservación (Esselman y Allan, 2011; Koleff et al., 2009; Sarkar y Margules, 2002).

Los patrones de distribución de la avifauna en la costa del Pacífico de Guerrero (CPGro) no han sido bien estudiados, aunque en el contexto estatal se ha señalado esta región como la segunda más importante por su riqueza de especies y su concentración de endemismos (Navarro, 1998). A la fecha, la información para esta región es dispersa y fragmentada, y no existen evaluaciones que prioricen su conservación bajo ningún enfoque. Por otra parte, los trabajos realizados han sido básicamente descriptivos y orientados principalmente a documentar ampliaciones de distribución de algunas especies (e. g., Davis, 1944; Torre, Martínez, Uribe y Santillán, 1979; Greene, Wilcove y Mcfarland, 1984; Navarro y Peterson, 1999). Sin embargo, en un estudio que involucró el oeste de México (Sonora y el oeste de Chihuahua a Chiapas), García-Trejo y Navarro-Sigüenza (2004) describieron los patrones de riqueza y endemismo a partir de datos primarios, y encontraron que la riqueza presenta tendencia a incrementarse hacia el sur, mientras que el endemismo se concentra en el centro de la región (Guerrero y Oaxaca). Ramírez-Bastida et al. (2008) analizaron los patrones de distribución de las aves acuáticas de México y obtuvieron que la mayor riqueza de estas especies (incluyendo residentes y migratorias) se concentra a lo largo de las costas. Así, la costa del Pacífico de México ha sido reconocida como una de las regiones con mayor riqueza y endemismo para las aves en México (e. g., García-Trejo y Navarro-Sigüenza, 2004; Navarro-Sigüenza et al., 2014; Peterson y Navarro-Sigüienza, 2000), por lo que los estudios sobre prioridades de conservación a lo largo de la región, y en particular en la CPGro, son de amplia importancia. Actualmente, la fauna de la región costera de Guerrero está amenazada debido al constante desarrollo de proyectos turísticos y actividades agrícolas (Meléndez-Herrada, Chimal, Figueroa, Vázquez y García, 2015), los cuales gradualmente sustituyen los remanentes de vegetación original.

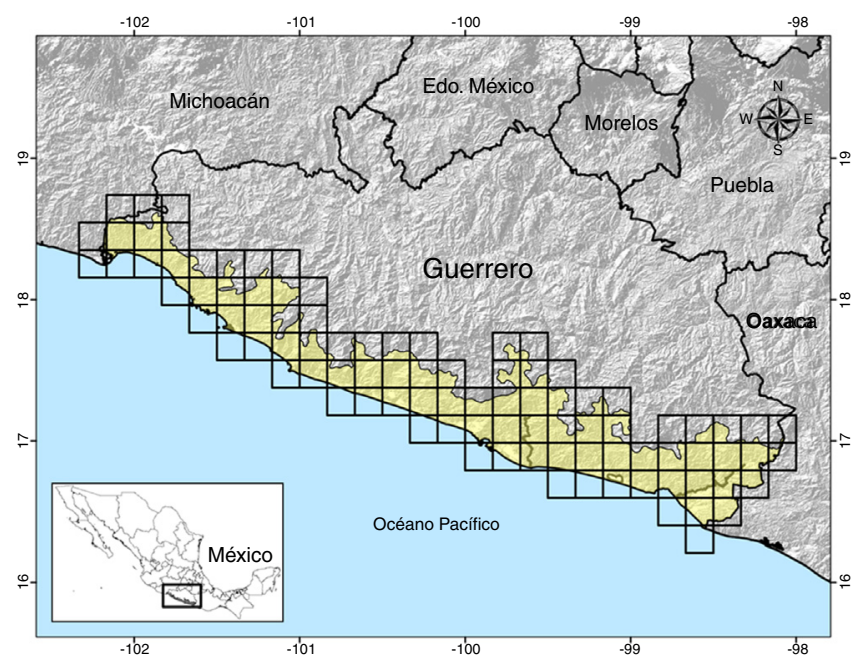

Figura 1. Ubicación geográfica de la costa del Pacífico de Guerrero. Se muestra la gradilla de $0.17^{\circ} \times 0.17^{\circ}\left(326 \mathrm{~km}^{2}\right)$ utilizada para los análisis de distribución e identificación de áreas para la conservación de la avifauna de la región.

En este trabajo se recopilaron datos puntuales sobre las aves en la CPGro, con los cuales se generaron MDE abarcando toda el área de distribución de cada especie. A partir de los MDE, se obtuvieron los patrones de distribución correspondientes a riqueza, endemismo y de especies en alguna categoría de riesgo. Los patrones obtenidos se emplearon para la propuesta de áreas de conservación para la avifauna sobre la base de 2 métodos de análisis: 1) complementariedad, y 2) PAE. Las áreas obtenidas en estos análisis se compararon con las ANP que se encuentran en la CPGro, con el fin de evaluar su papel como instrumentos reales de conservación. Adicionalmente, se usaron las Áreas de Importancia para la Conservación de las Aves (AICA) para evaluar su traslape geográfico con las áreas determinadas en este estudio.

\section{Materiales y métodos}

La CPGro se ubica entre los paralelos $16^{\circ} 02^{\prime} 01^{\prime \prime}$ y $18^{\circ} 08^{\prime} 59^{\prime \prime}$ $\mathrm{N}$, y en los meridianos $98^{\circ} 05^{\prime} 45^{\prime \prime}$ y $102^{\circ} 15^{\prime} 30^{\prime \prime} \mathrm{O}$. El área de estudio abarca una superficie de $15,026 \mathrm{~km}^{2}$, lo que representa el $23.6 \%$ del territorio del estado de Guerrero $\left(63,621 \mathrm{~km}^{2}\right.$; Semarnat, 2014). Colinda al sur con el océano Pacífico, al norte con las montañas de la sierra Madre del Sur, al este con las costas del estado de Oaxaca y al oeste con las costas de Michoacán (fig. 1). La altitud de la región va desde el nivel del mar hasta pequeñas áreas con elevaciones de hasta 2,000 m snm.

Se obtuvieron datos primarios (presencia) para toda el área de distribución de cada una de las especies de aves registradas en la zona de estudio en el periodo 1920-2009 a partir de 4 fuentes principales: 1) consultas a la base de datos del Atlas de las aves de México (Navarro, Peterson y Gordillo-Martínez, 2003), que contiene registros de ejemplares de aves mexicanas depositados en distintas colecciones del mundo; 2) registros observacionales del portal web de aVerAves (http://www.averaves.org/); 3) trabajo de campo en diferentes localidades de la costa de Guerrero realizado durante un periodo de 10 años (hasta 2009), y 4) consultas a la literatura científica para el área de estudio (e. g., 
Navarro y Peterson, 1999; Peterson, Rice y Navarro-Sigüenza, 2004). Con esta información se construyó una base de datos que incluyó 335,664 registros y a partir de la cual se realizaron consultas y extracción de información relevante para los objetivos de este estudio. Del total de especies, la mayoría (361 especies) presenta registros recientes de los últimos 10 años (a partir de 1999) y para 9 especies solo se encontraron registros anteriores a esa década (e. g., Colinus coyolcos, Cyrtonyx sallei, Gallinago gallinago, Onychoprion anaethetus, Icterus dickeyae). La base incluyó campos con la siguiente información: nombre científico, categorías supraespecíficas como orden y familia, estatus de endemismo (Peterson y Navarro-Sigüenza, 2000), categoría de residencia (Howell y Webb, 1995) y la categoría de riesgo de acuerdo con la NOM-059-SEMARNAT-2010 (Semarnat, 2010).

Para este estudio, la taxonomía utilizada a nivel de especie siguió la propuesta de Navarro-Sigüienza y Peterson (2004), basada en el concepto filogenético/evolutivo de especie. Esta propuesta se prefirió debido a que, en términos de conservación, podría evitar la subestimación de linajes bien diferenciados que en otras propuestas han sido considerados poblaciones de especies ampliamente distribuidas (García-Trejo y NavarroSigüenza, 2004; Navarro-Sigüenza y Peterson, 2004; Rojas-Soto et al., 2003; Rojas-Soto, Navarro-Sigüienza y Espinosa-de los Monteros, 2009). Las categorías supraespecíficas siguen lo propuesto por la AOU (1998) y sus adendas (www.aou.org).

Se evaluó la riqueza de especies de las aves de la zona de estudio con el uso del estimador de cobertura basado en incidencia (estimador ICE), usando cuadrantes de $0.17^{\circ}$ por lado, los cuales se prefirieron luego de realizarse comparaciones con otros tamaños de celda sin encontrar diferencias claras en los análisis de acumulación de especies y de identificación de áreas de endemismo y de complementariedad. Por otra parte, el estimador ICE basa su cálculo en la estimación de la cobertura de la muestra - i. e., la proporción de la riqueza representada en un conjunto de muestras de incidencia replicados; Colwell y Coddington (1994), Gotelli y Colwell (2011)_, por lo que es insensible al tamaño de muestra (Chazdon, Colwell, Denslow y Guariguata, 1998; Hortal, Borges y Gaspar, 2006). El análisis se realizó empleando el programa EstimateS v. 8 (Colwell, 2006), mediante 100 repeticiones aleatorias del orden de las muestras, lo que permite eliminar la influencia que tiene el orden en el cual estas se adicionan al total (Moreno y Halffter, 2000).

Para la elaboración de los MDE de las aves de la región se utilizó el algoritmo genético para la producción de conjuntos de reglas (GARP, por sus siglas en inglés; Stockwell y Noble, 1992; Stockwell y Peters, 1999), el cual ha sido ampliamente usado para el entendimiento de la distribución geográfica y ecológica de las especies (e. g., Peterson, 2001; Peterson, Papeş y Eaton, 2007; Tsoar, Allouche, Steinitz, Rotem y Kadmon, 2007). GARP trabaja mediante un proceso iterativo (generaciones) a través de la formación de reglas usando características inferenciales (atómicas, de rango, de rango negado y regresión logística), que son seleccionadas, evaluadas, probadas y aprobadas para pasar o no a la siguiente generación. El proceso de selección de reglas se termina cuando se alcanza un número limitado de iteraciones (máximo 1,000 iteraciones) o cuando existe un límite de convergencia (en este caso de 0.01), lo que establece una condición para detener las iteraciones cuando las nuevas reglas no mejoran el modelo (Stockwell y Noble, 1992; Stockwell y Peters, 1999).

Los modelos de distribución se generaron con el uso de 19 variables climáticas propuestas por Cuervo-Robayo et al. (2013), que son resultado de la interpolación del promedio de registros climáticos para México de 1910 a 2009. Adicionalmente, se consideraron 3 coberturas topográficas (elevación, pendiente e índice topográfico) del U.S. Geological Survey Hydro 1k (http://edcdaac.usgs.gov/gtopo30/hydro). Todas las variables se remuestrearon a una resolución espacial de $1 \mathrm{~km}^{2}$.

Para obtener una mejor caracterización de las áreas de idoneidad ambiental de las especies de aves registradas en la CPGro, los MDE se generaron sobre la base del total de registros disponibles para toda el área de distribución de cada especie. Todos los registros estuvieron acotados al intervalo temporal (1910-2009) cubierto por las variables bioclimáticas utilizadas. Aunque la mayoría de las especies tuvieron un alto número de registros (> $1,000)$, algunas especies contaron con menos de 50 registros (e. g., Nomonyx dominicus, C. coyolcos, C. sallei, O. anaethetus, Setophaga nigrilora), por lo que se dividieron en 2 grupos para la obtención de los MDE: 1) especies con más de 50 registros, y 2) especies $>1$ y $<50$ registros (véase el apéndice). Para la elaboración de los MDE se realizaron 100 réplicas por especie. La evaluación de los MDE con más de 50 registros (356 especies) se llevó a cabo usando pruebas extrínsecas, las cuales consisten en dividir el total de registros en datos de entrenamiento y validación, para lo cual se utilizó una proporción de $70 \%-30 \%$, respectivamente. Los modelos de especies con $>1$ y $<50$ registros (14 especies) se validaron utilizando evaluación intrínseca calculada directamente por el algoritmo (Stockwell y Peters, 1999). Esta evaluación consiste en utilizar el 100\% de los registros para entrenamiento y GARP genera puntos aleatorios para su validación (Stockwell y Peters, 1999). De las 100 réplicas obtenidas para cada especie, se eligieron los 10 mejores modelos sobre la base de los menores errores de omisión y valores de comisión, cercanos a la mediana del porcentaje del área predicha (Anderson, Lew y Peterson, 2003). Estos modelos fueron sumados para obtener un modelo final de consenso que incluyera al menos el $90 \%$ de los registros de cada especie. Los mapas finales de cada especie fueron sumados, lo que permitió la obtención de los patrones de riqueza, endemismo y especies en estatus de riesgo de la avifauna del área de estudio.

El análisis de complementariedad permite identificar el número mínimo de áreas que contienen la máxima diversidad biológica, lo que en la actualidad representa un requisito fundamental para la conservación (Humphries, van Wright y Williams, 1991; Urquiza-Haas y Alarcón, 2011; Vane-Wright et al., 1991). Para este análisis se utilizó la misma gradilla compuesta de 95 celdas de $0.17^{\circ}$ por lado (326 km²; fig. 1) empleada en los análisis anteriores (véase Métodos). Se utilizaron los MDE obtenidos para el total de la avifauna del área de estudio y se elaboró una matriz básica de datos (MBD) de presencia-ausencia de las especies en cada celda de la gradilla, en la cual se codificó con «1» aquellas celdas que incluyeran área predicha y con «0» donde hubo ausencia de áreas potenciales. Debido a que la biología 


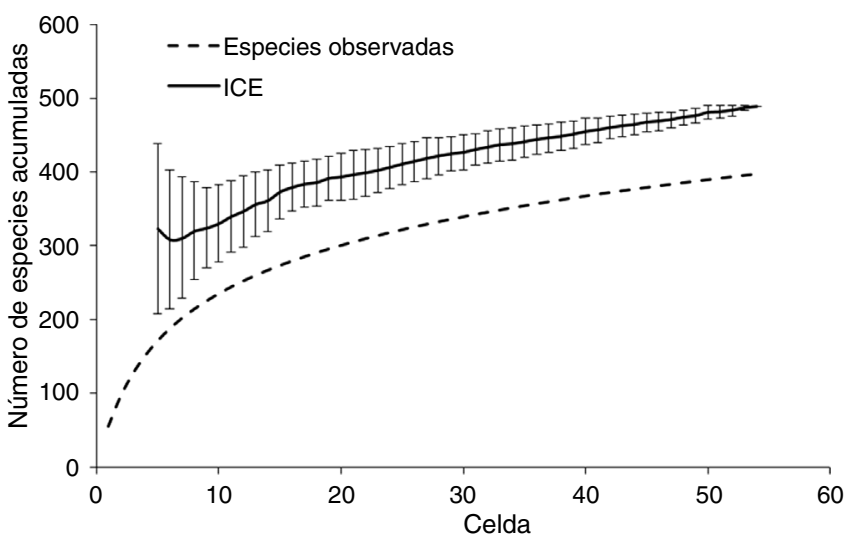

Figura 2. Curva de acumulación de especies de la avifauna de la costa del Pacífico de Guerrero. Las líneas verticales representan la desviación estándar para cada valor estimado por muestra (celdas de $0.17^{\circ}$ por lado).

y los patrones de distribución de las especies terrestres suelen ser diferentes a las acuáticas (Wiens, 1989), se llevaron a cabo análisis de complementariedad separados, que incluyeron 265 especies de aves terrestres y 105 especies de aves acuáticas. Los análisis de complementariedad se realizaron a través de un proceso iterativo, en el cual primero se seleccionó la celda con la mayor riqueza de especies; después, se eligió una segunda celda con la mayor concentración de especies no incluidas en la primera (Venegas-Barrera y Manjarrez, 2011) y así, de manera sucesiva, hasta identificar el número mínimo de celdas complementarias que en conjunto albergaran el $100 \%$ de la riqueza avifaunística de la región. Se desplegaron las ANP y las AICA (Arizmendi y Márquez-Valdelamar, 2000) sobre las áreas de conservación identificadas en este análisis con el fin de evaluar el traslape geográfico.

El método PAE permitió identificar áreas de endemismo para la avifauna de la CPGro sobre la base de las sinapomorfías geográficas (taxones endémicos a cada grupo de celdas; Escalante y Morrone, 2003; Morrone y Crisci, 1995). Estas sinapomorfías representan especies exclusivas, son irremplazables y, por lo tanto, altamente importantes para la conservación (MéndezLarios et al., 2005; Rovito et al., 2004). Cabe resaltar que el PAE puede ser interpretado dinámica o estáticamente (Nihei, 2006). El PAE dinámico analiza los cambios distribucionales a través del tiempo y el PAE estático se refiere a la identificación de homologías biogeográficas primarias en un único horizonte geológico (Escalante, 2011); es decir, es útil en la identificación de patrones y no necesariamente supone procesos. De acuerdo con estas definiciones, en este estudio se utilizó el PAE en un contexto estático o descriptivo (Escalante, 2011; Nihei, 2006), por lo que las áreas de endemismo identificadas en este estudio tienen un sentido ecológico y no histórico.

Debido a que la distribución de las aves migratorias y acuáticas obedece a patrones históricos y ecológicos diferentes con relación a las residentes terrestres (Komar, 2003), este análisis solo se aplicó a la avifauna residente terrestre cuyos MDE se generaron previamente (181 especies en total). Por tal razón, se generó otra MBD para el PAE, la cual incluyó un área hipotética

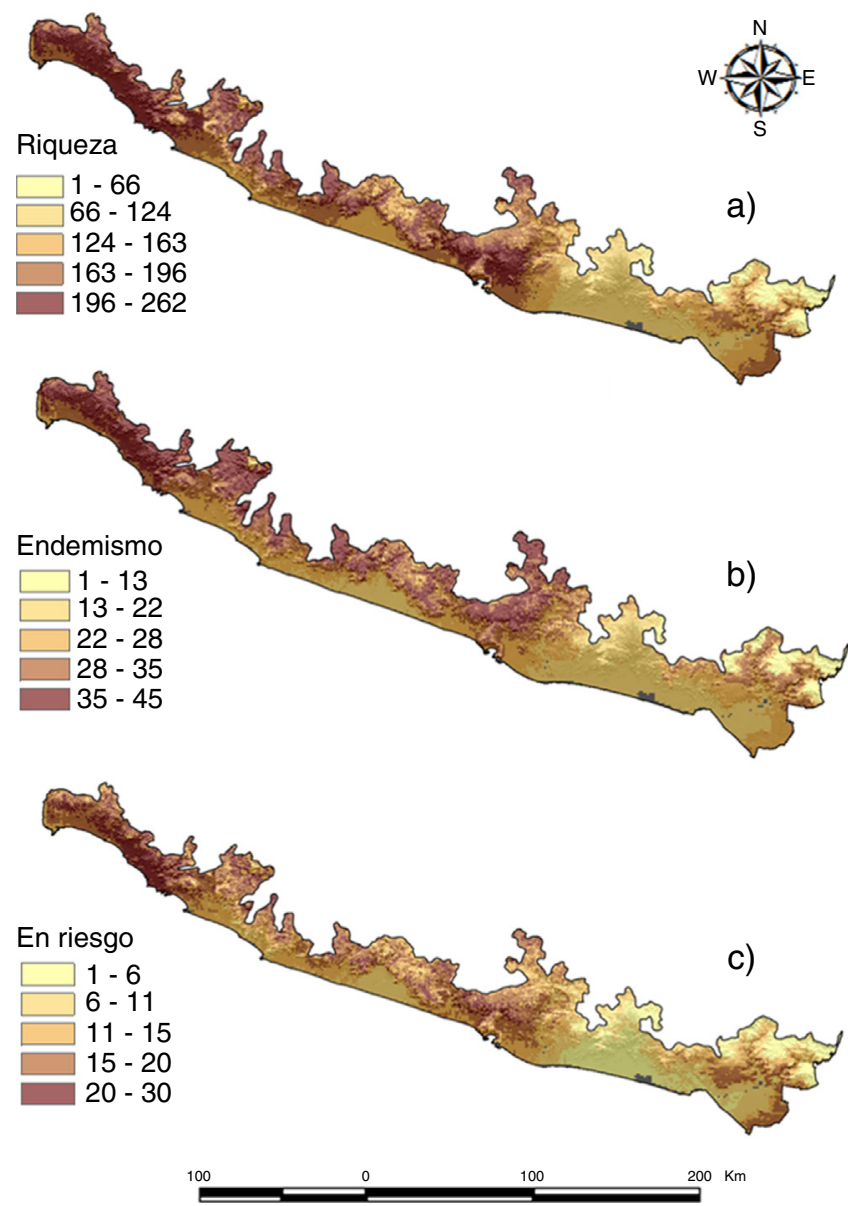

Figura 3. Patrones de riqueza (a), endemismo (b) y especies en riesgo (c) de las aves de la costa del Pacífico de Guerrero estimados a partir de la suma de los MDE individuales.

con «0» para enraizar los cladogramas (Morrone, 1994). Este análisis se ejecutó con las 95 celdas bajo las mismas dimensiones del ejercicio anterior $\left(0.17^{\circ} \times 0.17^{\circ}\right)$. La obtención de los cladogramas para este análisis se llevó a cabo con el programa Nona ver. 2.0 (Goloboff, Farris y Nixon, 2000) en WinClada ver. 1.00.08 (Nixon, 1999), a partir de una búsqueda heurística con 1,000 réplicas de adición de secuencias al azar, intercambio de ramas por tree bisection and reconnection (TBR) y se retuvieron 10 cladogramas por réplica. Se obtuvo un cladograma final de consenso estricto y se calcularon los índices de consistencia (CI) y de retención (RI). Para estimar el valor de soporte de las ramas seleccionadas se realizó una prueba de bootstrap con 500 réplicas (Morrone, 2003). Las áreas resultantes del PAE se compararon con las áreas obtenidas a partir del análisis de complementariedad y aquellas que resultaron congruentes en ambos análisis se consideraron con un nivel de prioridad de conservación mayor.

\section{Resultados}

\section{Patrones de distribución geográfica de las especies}

La riqueza de la CPGro está compuesta por 370 especies incluidas en 58 familias y 21 órdenes; de estas, un total de 
51 especies son endémicas de México, mientras que el estatus estacional está repartido en 216 especies residentes $(58 \%)$ y 154 migratorias (42\%; apéndice). El estimador de riqueza ICE indicó que se tiene registrado el $77 \%$ del total esperado (481 especies \pm 0.07 ; DE); es decir, se estima que faltan alrededor de 114 especies por registrar en el área de estudio (fig. 2). Estos valores indican que el inventario para la zona es representativo (Gómez-de Silva y Medellín, 2001). Los patrones de riqueza estimados a partir de los MDE (fig. 3 a) indican que las áreas de mayor riqueza de especies (196-262 especies), endemismo (35-45 especies) y especies en riesgo (20-30 especies) se ubican principalmente hacia las zonas montañosas del centro-oeste del área de estudio y disminuyen hacia la porción oriental (fig. 3 $\mathrm{a}-\mathrm{c})$.

\section{Análisis de complementariedad}

El análisis de complementariedad para la avifauna terrestre mostró que de las 95 celdas en las que se dividió el área de estudio, un total de 6 albergan el 100\% de la riqueza estimada a partir de los MDE (265 especies; fig. 4 a). Estas celdas representan el $17 \%\left(2,512 \mathrm{~km}^{2}\right)$ de la superficie total de la CPGro $\left(15,026 \mathrm{~km}^{2}\right)$. En la primera celda (37) existe el $95.5 \%$ de la avifauna terrestre modelada en el área de estudio y al localizar las 2 celdas subsecuentes (27 y 50) el porcentaje se incrementó hasta el $99 \%$ de la riqueza total (tabla 1). La mayor cantidad de especies terrestres endémicas y en riesgo para el área de estudio se encontró en la celda 37 (tabla 1). Para la avifauna acuática (fig. 4 b), 6 celdas $(13,52,17,4,21,34)$ acumularon el $100 \%$ de las especies modeladas (tabla 1); en particular, las celdas 13 y 52 acumularon al total de especies acuáticas en alguna categoría de riesgo. Al sobrelapar las ANP de la franja costera de Guerrero (Reserva Estatal El Limón y Parque Nacional El Veladero), se observa congruencia geográfica total con las áreas complementarias identificadas en este análisis (fig. 4 c). Por otra parte, las AICA mostraron menor coincidencia con las áreas complementarias (fig. 4 c). Del total de superficie de las áreas o celdas complementarias en este estudio, el $9 \%$ se traslapa con alguna de las AICA.

\section{Análisis de parsimonia de endemismos}

Los resultados del PAE generaron 1,000 cladogramas, de los cuales se obtuvo uno de consenso estricto de 834 pasos (CI: 20, RI: 76) con un soporte de ramas de entre el 88 y el $100 \%$ de acuerdo con la prueba de bootstrap (fig. 5 a). El cladograma de consenso estricto presentó altos niveles de homoplasia, lo que generó algunas politomías (fig. 5 a); sin embargo, se identificaron 2 áreas de endemismo definidas por al menos 2 taxones (tabla 2). La primera está representada por las celdas 4, 5, 7, 8, 10-12 y 15, y la segunda por las celdas $34,39,40$ y 47 (fig. 5 b). De estas, la celda 4 también fue identificada en el análisis de complementariedad de las aves terrestres y acuáticas (fig. 4 a y b). Las áreas de endemismo determinadas incluyeron el $91 \%$ de la avifauna total modelada (370 especies).

\section{Discusión}

La riqueza avifaunística de la CPGro (370 especies) representa el $68 \%$ de lo registrado para Guerrero (545 especies; Almazán-Núñez, Meléndez-Herrada, García-Vega, Sierra-Morales y Méndez-Bahena, 2017) y el 35\% de la avifauna nacional (Navarro-Sigüenza et al., 2014). Adicionalmente, la riqueza de especies reportada en este estudio aumenta en un $24 \%$ la previamente registrada por Navarro (1998) para la planicie costera del estado de Guerrero. Por citar algunos ejemplos, durante los últimos 5 años se ha documentado la presencia de Megascops seductus, Rhodinocichla schistacea y Setophaga citrina (Almazán-Núñez et al., 2017; Peterson et al., 2004). Este aumento puede ser un reflejo del interés que en los últimos años ha tenido la comunidad científica y aficionada en el reconocimiento de avifaunas regionales poco conocidas, y cuyos datos son distribuidos a través de herramientas digitalizadas disponibles en páginas web (Gómez-de Silva y Alvarado, 2010; Navarro et al., 2003). Por otra parte, la elevada riqueza del área de estudio cobra más relevancia si se considera que la extensión territorial de la CPGro ocupa aproximadamente la cuarta parte del estado $\left(24 \% ; 15,026 \mathrm{~km}^{2}\right)$. Estudios futuros podrían estar dirigidos a confirmar la presencia de las especies incluidas en este estudio, debido a los constantes cambios en el uso del suelo o bien, a ampliar el listado de acuerdo con el valor esperado por el estimador de riqueza utilizado. Por otra parte, la riqueza de la región de estudio es resultado de las condiciones ecológicas a lo largo del gradiente altitudinal que va desde el nivel del mar hasta los 2,000 m, y por la presencia de tipos de vegetación como la selva baja caducifolia, selva mediana subcaducifolia, bosques húmedos de montaña y manglares, que definen local y regionalmente la diversidad de especies (Carreto-Pérez, Almazán-Juárez, Sierra-Morales y AlmazánNúñez, 2015; García-Trejo y Navarro-Sigüenza, 2004). Por ejemplo, es posible encontrar en el área de estudio especies características de laderas de montaña como Cyanocorax speciosa, Myadestes occidentalis, Amazilia viridifrons, Hylocharis leucotis, Melanotis caerulescens, Melanerpes formicivorus, Micrastur semitorquatus y Piranga hepatica. Asimismo, existen ambientes lacustres que albergan una alta diversidad de aves acuáticas y marinas (e. g., Anatidae, Chadriformes; MeléndezHerrada et al., 2015; Ramírez-Bastida et al., 2008), además de un importante número de especies de aves migratorias de invierno. De hecho, para el componente migratorio (de paso o de permanencia invernal), esta región constituye una de las áreas y/o rutas más importantes para las aves migratorias neotropicales (Hutto, 1995; Ramírez-Bastida et al., 2008).

La mayor riqueza potencial de aves en la CPGro está concentrada en la porción centro-oeste del área de estudio (abarcando una superficie de $3,294 \mathrm{~km}^{2}$ ), desde la cuenca del río Papagayo hasta Zihuatanejo (celdas 13, 14, 17, 52, 58), particularmente en pequeños sistemas montañosos donde predomina la selva baja caducifolia, la selva mediana subcaducifolia y el bosque mesófilo de montaña, ecosistemas reconocidos por su diversidad biológica (Dirzo, Young, Mooney y Ceballos, 2011; Lara-Lara et al., 2008; Meléndez-Herrada et al., 2015; Villaseñor y GualDíaz, 2014). También comprende zonas ubicadas a nivel del 


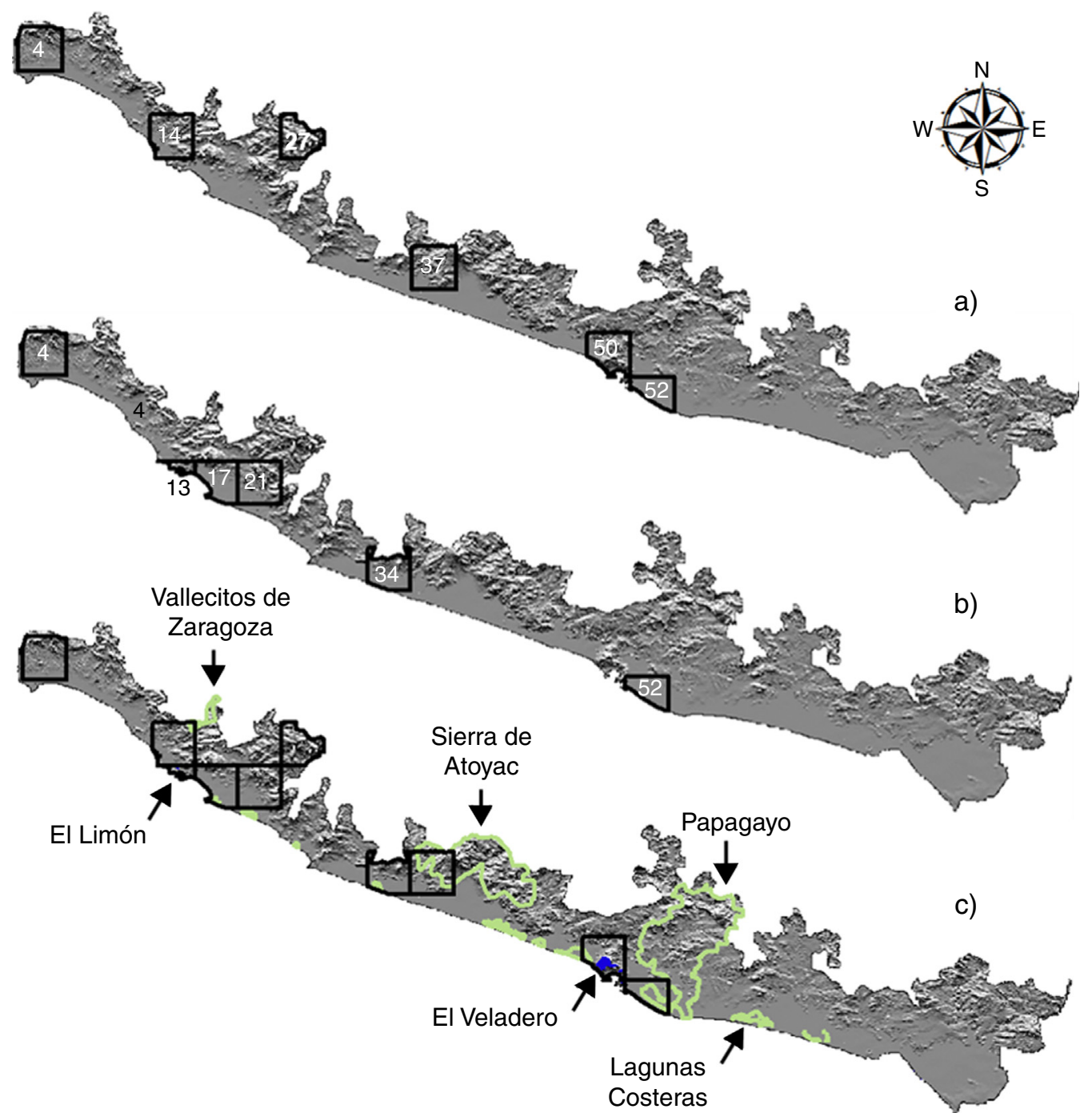

Figura 4. Áreas complementarias identificadas a partir de la distribución potencial de las aves (a) terrestres, (b) acuáticas y (c) traslape con las ANP (polígonos azules) y AICA (polígonos verdes) en la costa del Pacífico de Guerrero.

Tabla 1

Número de especies complementarias para las aves terrestres y acuáticas, especies endémicas y en riesgo encontradas en las celdas seleccionadas por el análisis de complementariedad.

\begin{tabular}{|c|c|c|c|c|c|}
\hline Número de celda & Especies complementarias & Especies en riesgo & Especies endémicas & Riqueza acumulada & Porcentaje \\
\hline 37 & 253 & 31 & 51 & 253 & 95.5 \\
\hline 27 & 7 & 1 & 0 & 260 & 98.1 \\
\hline 4 & 1 & 0 & 0 & 263 & 99.2 \\
\hline 14 & 1 & 0 & 0 & 264 & 99.6 \\
\hline 52 & 1 & 0 & 0 & 265 & 100 \\
\hline 13 & 86 & 14 & 0 & 86 & 82.0 \\
\hline 52 & 13 & 3 & 0 & 99 & 94.3 \\
\hline 17 & 3 & 0 & 0 & 102 & 97.1 \\
\hline 4 & 1 & 0 & 0 & 103 & 98.1 \\
\hline 21 & 1 & 0 & 0 & 104 & 99.0 \\
\hline 34 & 1 & 0 & 0 & 105 & 100 \\
\hline
\end{tabular}




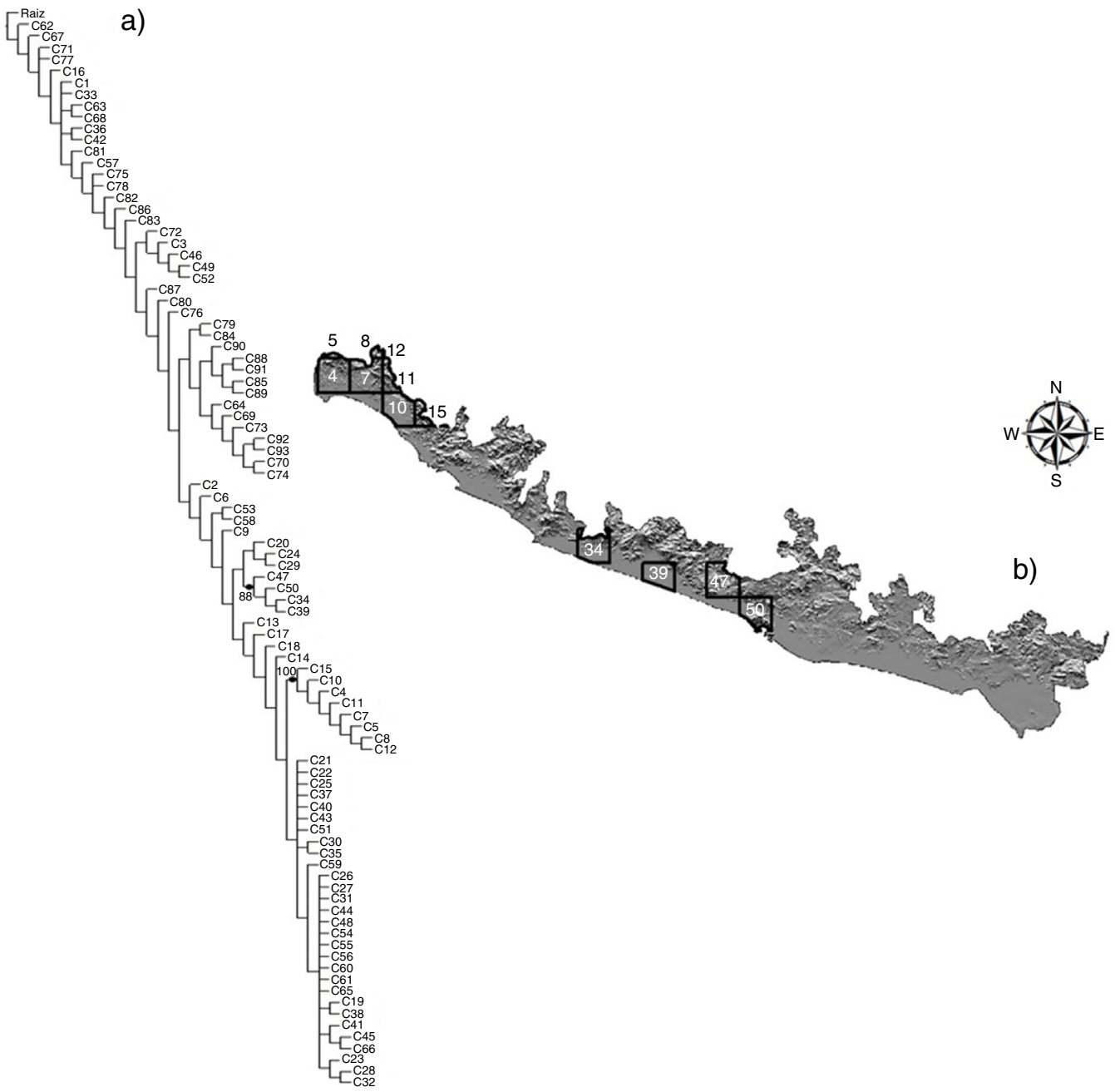

Figura 5. Análisis de parsimonia de endemismos (a) y correspondencias geográficas (b) sobre el mapa de la costa del Pacífico de Guerrero. Los números en los nodos corresponden al valor de confiabilidad bootstrap.

- Taxones sinapomórficos (véase la tabla 2).

mar con presencia de algunos sistemas lacustres en asociación con manglares, particularmente en la porción más occidental del área de estudio (Zihuatanejo, laguna de Potosí). Por otro lado, aunque la CPGro no es la zona con mayor número de endemismos en Guerrero en comparación con la sierra Madre del Sur (Navarro-Sigüenza et al., 2016) y la cuenca del Balsas (Feria-Arroyo y Peterson, 2002), presenta un alto número de especies endémicas (Navarro, 1998; Peterson y NavarroSigüenza, 2000). De acuerdo con la propuesta taxonómica de Peterson y Navarro-Sigüenza (2000), algunas de estas son endémicas de las tierras bajas del sur, región en la que se encuentra el área de estudio (e. g., Amazona finschi, Piaya mexicana, Glaucidium palmarum, Phaethornis mexicanus, Trogon citreolus, Attila pacificus, Pachyramphus albiventris, Cyanocorax sanblasianus, Arremonops sumichrasti). La distribución de las especies endémicas presentó en general el mismo patrón que el de la riqueza, en el cual se observa una mayor correspondencia con las zonas montañosas del área de estudio. Esto confirma parcialmente el hecho de que el endemismo está asociado de manera primaria a laderas montañosas (Escalante, Navarro y Peterson, 1998; García-Trejo y Navarro-Sigüenza, 2004; Navarro-Sigüenza et al., 2014; Peterson y Navarro-Sigüenza, 2000), como lo sugiere la presencia de A. viridifrons, A. finschi, G. palmarum, Xenotriccus mexicanus, T. citreolus, Granatellus venustus, A. sumichrasti y Trhyophilus sinaloa. Existen otras especies endémicas en la porción oeste de la CPGrO asociadas principalmente a la franja costera (i. e., Deltarhynchus flammulatus, $R$. schistacea, Passerina leclancherii, C. sanblasianus). El número de especies en riesgo aumenta ligeramente hacia la franja costera, donde el desarrollo acelerado de las actividades económicas ha propiciado un crecimiento desordenado en las costas y algunas de sus especies se han extinto localmente, como ocurrió con la guacamaya verde (A. militaris) en el declive costero de Guerrero (Almazán-Núñez y Nova-Muñoz, 2006) y de Michoacán (Monterrubio-Rico, Labra-Hernández, OrtegaRodríguez, Cancino-Murillo y Villaseñor-Gómez, 2011).

Los análisis de complementariedad, tanto para la avifauna terrestre como acuática, identificaron 6 celdas (de un total de 
95) en la CPGro como prioritarias para la conservación, lo que representa una extensión territorial de $2,513 \mathrm{~km}^{2}$, equivalente al $17 \%$ de la superficie del área. Estas celdas incluyen áreas con alta riqueza, así como algunas zonas previamente identificadas por su alta diversidad avifaunística, como los sistemas lacustres a nivel del mar (Arizmendi y Márquez-Valdelamar, 2000; Meléndez-Herrada et al., 2015) y las zonas montañosas del declive costero de la sierra de Atoyac (Navarro, 1992). Esta última área en particular ha sido reconocida además por su alta diversidad en otros grupos faunísticos y florísticos (Monteagudo y Luis, 2013; Ponce-Ulloa y Llorente-Bousquets, 1993) y por su valor paisajístico (Luna-Vega, Espinosa y Contreras-Medina, 2016).

De las 6 celdas identificadas como complementarias para las aves terrestres y acuáticas, solo 2 ( 4 y 52) coincidieron en ambos análisis. Esto demuestra la diferencia en los hábitos y distribución de cada grupo y, por otro lado, confirma la importancia de estas áreas para especies de relevancia para la conservación (i. e., A. oratrix; Monterrubio-Rico et al., 2016). Por otro parte, si bien el traslape geográfico de estas áreas complementarias con las ANP fue total, nuestros análisis sugieren que estas áreas de conservación oficiales pueden ser ampliadas para incluir un mayor número de especies, así como para facilitar la conectividad entre distintas poblaciones (Bélisle, 2005; Haig, Mehlman y Oring, 1998).

El análisis con las ANP mostró un traslape total con las áreas identificadas por el análisis de complementariedad y que resultó menor con las AICA (9\%). Existen celdas particularmente en el centro-oeste del área de estudio que prácticamente no presentan sobrelapamiento con ninguna de las ANP declaradas o las AICA propuestas, aun cuando el valor de complementariedad (diversidad total) e irremplazabilidad (especies restringidas) es alto (figs. 4 y 5). Así por ejemplo, aunque las celdas 27 y 37 presentaron la más alta complementariedad de especies terrestres de toda la CPGro, no existe coincidencia con ninguna de las ANP y solo la celda 27 tiene un traslape del $55 \%$ con el AICA de la sierra de Atoyac. Con excepción de las celdas 4, 34 y 50, las 2 áreas definidas por el PAE (i. e., área 1: celdas 4, 5, 7, 8, 10, $11,12,15$; área 2 : celdas $34,39,47,50$ ) difieren de las determinadas por el análisis de complementariedad. Dado que el PAE se basa principalmente en especies compartidas por un conjunto de áreas (sinapomorfías) sin hacer referencia a las especies totales, no consideró en sus resultados celdas identificadas como complementarias (i. e., 13, 14, 17, 21, 27, 37, 52). Debido a estas diferencias, los resultados de ambos análisis fueron interpretados de manera complementaria. Así, las celdas identificadas en ambos análisis representan un nivel de priorización mayor que el resto, dado que están definidas por el número de especies endémicas y por la alta riqueza específica a nivel local. En particular, las áreas propuestas con el PAE representan sitios importantes para la conservación donde la presencia de las especies con distribución local y restringida hace que estas áreas sean únicas, y por lo tanto irremplazables, un criterio importante en la definición de zonas prioritarias para la conservación (Méndez-Larios et al., 2005; Zamora-Manzur et al., 2011).

Aunque el gradiente altitudinal de la región es amplio (0-2,000 m snm), las áreas de endemismo presentan pocas varia-
Tabla 2

Áreas de endemismo (celdas) y especies que sustentan los clados.

\begin{tabular}{ll}
\hline Celdas & Especies \\
\hline $4,5,7,8,10-12,15$ & Basileuterus culicivorus, B. lachrymosa, Cyrtonyx sallei \\
$34,39,47,50$ & Phaethornis mexicanus, Cyanocorax speciosa \\
\hline
\end{tabular}

ciones climáticas. Por ejemplo, en las celdas correspondientes a la primer área $(4,5,7,8,10-12,15)$ predominan los climas cálidos semisecos y subhúmedos a lo largo de un intervalo altitudinal que va desde el nivel del mar hasta los 1,300 m, lo que explica la presencia de sinapomorfías geográficas características de laderas montañosas del declive costero de la sierra Madre del Sur, como Basileuterus culicivorus, B. lachrymosa y C. sallei (Howell y Webb, 1995). En la segunda área de endemismo (celdas 34, 39, 47 y 50), se presenta un gradiente altitudinal similar con elevaciones hasta los 1,650 m snm (celda 47), por lo que el clima predominante pertenece a los cálidos semisecos y subhúmedos, excepto la celda 39, que presenta un clima semiárido cálido. Estas celdas están también sustentadas por especies características de laderas montañosas semihúmedas ( $P$. mexicanus, $C$. speciosa). Algunas de las especies que apoyan los clados en las 2 áreas identificadas representan poblaciones diferenciadas a las del resto del país (C. sallei, C. speciosa, P. mexicanus), cuya distribución comprende el declive montañoso del Pacífico, particularmente de los estados de Guerrero y Oaxaca (Navarro-Sigüenza y Peterson, 2004), una región altamente importante por su nivel de endemismos (Navarro-Sigüenza et al., 2016).

La irregularidad en los datos puntuales que representan la diversidad avifaunística en cada celda puede deberse a las diferencias en el esfuerzo de muestreo, lo que indica que algunas áreas han sido temporal y espacialmente mejor muestreadas (Peterson, Navarro-Sigüenza y Benítez-Díaz, 1998), como ocurre en las cercanías de Acapulco, Zihuatanejo y Atoyac de Álvarez. Este aspecto pudo también haber influido en los patrones de riqueza encontrados. Por otra parte, en el PAE, las politomías pueden ser consideradas principalmente evidencia de una falta de esfuerzo de muestreo entre zonas adyacentes cuando se emplean datos primarios (Navarro, Liria, Piñango y Barrera, 2007; Zamora-Manzur et al., 2011), aunque se ha indicado que también podrían representar regiones con gradientes ecológicos o zonas de transición. Adicionalmente, podrían explicar las áreas con conexión histórica que pudieron sufrir procesos vicariantes tanto naturales (a partir de los datos de los MDE) como antrópicos (con los datos puntuales), generando extinciones locales dentro de la región de estudio. En particular, la degradación por cambios en el uso de suelo con fines urbanos, agrícolas y/o forestales, podría explicar vacíos y baja diversidad en algunas celdas. Esta desigualdad en los datos puede ser suplida por la extrapolación realizada en este estudio con los MDE.

Este estudio se enfocó en la identificación de zonas prioritarias para la avifauna en la CPGro, la cual ha sufrido importantes modificaciones por la acelerada pérdida de vegetación natural ocasionada por las actividades humanas (Peralta-Gómez, Diego- 
Pérez y Gual-Díaz, 2000). Por lo anterior, los análisis de los patrones de distribución, así como la evaluación de áreas de conservación propuestas en este estudio, pueden constituir una línea base para el establecimiento de prioridades de conservación, sin que ello garantice los procesos y la viabilidad de las poblaciones de aves, particularmente, cuando el sistema de ANP en el estado está pobremente representado (Bezaury-Creel et al., 2012; Koleff y Moreno, 2006), y probablemente tendría una eficiencia limitada en la protección de la avifauna regional. No obstante, recientemente se han llevado a cabo esfuerzos a nivel estatal para impulsar la creación de pequeñas reservas estatales con fines de conservación a lo largo del estado, incluida la CPGro (DOF, 2011). Un ejemplo de ello es la reciente creación de 6 parques estatales; sin embargo, cubren apenas el $0.06 \%$ de la superficie de la entidad (DOF, 2010, 2011, 2012). Por ello, y ante los constantes cambios en el uso del suelo y las alteraciones por cambio climático, resulta fundamental para esta región, y en particular para un estado rico en recursos biológicos como Guerrero, fomentar estrategias adicionales de conservación, como los corredores biológicos y otras iniciativas de conservación in situ que promuevan el uso sustentable de la biodiversidad regional. Se espera que los resultados de este estudio sirvan como base para futuras investigaciones que evalúen y comparen áreas de conservación para otros grupos taxonómicos y, además, contribuyan en la planeación sistemática de la conservación de esta región.

\section{Agradecimientos}

Los autores agradecen a Pablo Sierra por su apoyo en diferentes etapas del proyecto. A Liliana Parra, Antonio Estrada y Génesis Benítez, por su apoyo en el trabajo de campo. Edson Álvarez apoyó en la construcción del apéndice. La Facultad de Ciencias Químico Biológicas y la Escuela Superior de Ciencias Ambientales de la Universidad Autónoma de Guerrero otorgaron apoyo para las actividades logísticas del proyecto. Agradecemos el apoyo de 2 revisores anónimos, quienes hicieron comentarios críticos que mejoraron sustancialmente la versión original de este documento.

\section{Apéndice.}

Lista de las especies de aves registradas en la costa del Pacífico del estado de Guerrero (CPGro) de acuerdo con las diferentes fuentes consultadas. Para cada especie se incluye el estatus de estacionalidad (RP: residente permanente, MI: migratorio de invierno, MV: migratorio de verano, T: transitorio, V: visitante ocasional), el estatus de endemismo (E: endémico en México) y la categoría de riesgo (Pr: sujeta a protección especial, A: amenazada, $P$ : en peligro de extinción). El número de registros para la generación de los MDE se asignó como sigue: 1: especies con más de 50 registros; 2: especies con $>1 \mathrm{y}<50$ registros

\begin{tabular}{|c|c|c|c|c|}
\hline $\begin{array}{l}\text { Nombre } \\
\text { científico }\end{array}$ & $\begin{array}{l}\text { Estacio- } \\
\text { nalidad }\end{array}$ & Endemismo & $\begin{array}{l}\text { Categoría } \\
\text { de riesgo }\end{array}$ & $\begin{array}{l}\text { Grupo de } \\
\text { acuerdo } \\
\text { con el } \\
\text { número de } \\
\text { registros } \\
\text { obtenidos } \\
\text { para los } \\
\text { MDE }\end{array}$ \\
\hline $\begin{array}{r}\text { Tachybaptus } \\
\text { dominicus }\end{array}$ & $\mathrm{RP}$ & & $\operatorname{Pr}$ & 1 \\
\hline $\begin{array}{c}\text { Podilymbus } \\
\text { podiceps }\end{array}$ & $\mathrm{RP}$ & & & 1 \\
\hline $\begin{array}{l}\text { Podiceps } \\
\quad \text { nigricollis }\end{array}$ & MI & & & 1 \\
\hline Ardenna pacifica & V & & A & 1 \\
\hline $\begin{array}{l}\text { Phaethon } \\
\text { aethereus }\end{array}$ & $\mathrm{V}$ & & A & 1 \\
\hline Sula dactylatra & V & & & 1 \\
\hline Sula nebouxii & V & & $\operatorname{Pr}$ & 1 \\
\hline Sula leucogaster & $\mathrm{V}$ & & & 1 \\
\hline Sula sula & $\mathrm{V}$ & & A & 1 \\
\hline $\begin{array}{l}\text { Pelecanus eryth- } \\
\text { rorhynchos }\end{array}$ & MI & & & 1 \\
\hline $\begin{array}{l}\text { Pelecanus } \\
\quad \text { occidentalis }\end{array}$ & $\mathrm{RP}$ & & & 1 \\
\hline $\begin{array}{c}\text { Phalacrocorax } \\
\text { brasilianus }\end{array}$ & $\mathrm{RP}$ & & & 1 \\
\hline Anhinga anhinga & RP & & & 1 \\
\hline $\begin{array}{l}\text { Fregata } \\
\quad \text { magnificens }\end{array}$ & $\mathrm{RP}$ & & & 1 \\
\hline $\begin{array}{l}\text { Botaurus } \\
\quad \text { lentiginosus }\end{array}$ & MI & & A & 1 \\
\hline $\begin{array}{l}\text { Butorides } \\
\quad \text { virescens }\end{array}$ & $\mathrm{RP}$ & & & 1 \\
\hline Ixobrychus exilis & RP & & $\operatorname{Pr}$ & 1 \\
\hline $\begin{array}{l}\text { Tigrisoma } \\
\text { mexicanum }\end{array}$ & RP & & $\operatorname{Pr}$ & 1 \\
\hline Ardea herodias & MI & & & 1 \\
\hline Ardea alba & MI & & & 1 \\
\hline Egretta thula & $\mathrm{RP}$ & & & 1 \\
\hline Egretta rufescens & $\mathrm{RP}$ & & $\operatorname{Pr}$ & 1 \\
\hline Egretta caerulea & $\mathrm{RP}$ & & & 1 \\
\hline Egretta tricolor & $\mathrm{RP}$ & & & 1 \\
\hline Bubulcus ibis & $\mathrm{RP}$ & & & 1 \\
\hline $\begin{array}{l}\text { Butorides } \\
\quad \text { virescens }\end{array}$ & $\mathrm{RP}$ & & & 1 \\
\hline $\begin{array}{l}\text { Nycticorax } \\
\text { nycticorax }\end{array}$ & MI & & & 1 \\
\hline $\begin{array}{c}\text { Nyctanassa } \\
\text { violacea }\end{array}$ & $\mathrm{RP}$ & & & 1 \\
\hline $\begin{array}{l}\text { Cochlearius } \\
\text { cochlearius }\end{array}$ & $\mathrm{RP}$ & & & 1 \\
\hline Eudocimus albus & $\mathrm{RP}$ & & & 1 \\
\hline Plegadis chihi & MI & & & 1 \\
\hline Platalea ajaja & MI & & & 1 \\
\hline $\begin{array}{l}\text { Mycteria } \\
\text { americana }\end{array}$ & MI & & $\operatorname{Pr}$ & 1 \\
\hline Coragyps atratus & $\mathrm{RP}$ & & & 1 \\
\hline Cathartes aura & $\mathrm{RP}$ & & & 1 \\
\hline $\begin{array}{r}\text { Dendrocygna } \\
\text { autumnalis }\end{array}$ & $\mathrm{RP}$ & & & 1 \\
\hline $\begin{array}{l}\text { Dendrocygna } \\
\text { bicolor }\end{array}$ & $\mathrm{RP}$ & & & 1 \\
\hline
\end{tabular}




\begin{tabular}{|c|c|c|c|c|c|c|c|c|c|}
\hline $\begin{array}{l}\text { Nombre } \\
\text { científico }\end{array}$ & $\begin{array}{l}\text { Estacio- } \\
\text { nalidad }\end{array}$ & Endemismo & $\begin{array}{l}\text { Categoría } \\
\text { de riesgo }\end{array}$ & $\begin{array}{l}\text { Grupo de } \\
\text { acuerdo } \\
\text { con el } \\
\text { número de } \\
\text { registros } \\
\text { obtenidos } \\
\text { para los } \\
\text { MDE }\end{array}$ & $\begin{array}{l}\text { Nombre } \\
\text { científico }\end{array}$ & $\begin{array}{l}\text { Estacio- } \\
\text { nalidad }\end{array}$ & Endemismo & $\begin{array}{l}\text { Categoría } \\
\text { de riesgo }\end{array}$ & $\begin{array}{l}\text { Grupo de } \\
\text { acuerdo } \\
\text { con el } \\
\text { número de } \\
\text { registros } \\
\text { obtenidos } \\
\text { para los } \\
\text { MDE }\end{array}$ \\
\hline Cairina moschata & $\mathrm{RP}$ & & $\mathrm{P}$ & 1 & Aramides & $\mathrm{RP}$ & & & 1 \\
\hline Anas acuta & MI & & & 1 & cajaneus & & & & \\
\hline Anas strepera & MI & & & 1 & Aramides axillaris & $\mathrm{RP}$ & & A & 1 \\
\hline Anas americana & MI & & & 1 & Porzana carolina & MI & & & 1 \\
\hline Anas discors & MI & & & 1 & Pardirallus & $\mathrm{RP}$ & & & 1 \\
\hline Anas cyanoptera & MI & & & 1 & maculatus & & & & \\
\hline Anas crecca & MI & & & 1 & Porphyrio & $\mathrm{RP}$ & & & 1 \\
\hline Anas clypeata & MI & & & 1 & martinicus & & & & \\
\hline Aythya americana & MI & & & 1 & Gallinula & MI & & & 1 \\
\hline Aythya affinis & MI & & & 1 & chloropus & & & & \\
\hline Nomonyx & $\mathrm{RP}$ & & A & 2 & Fulica americana & MI & & & 1 \\
\hline dominicus & & & & & Pluvialis & MI & & & 1 \\
\hline Oxyura & MI & & & 1 & squatarola & & & & \\
\hline jamaicensis & & & & & Charadrius & $\mathrm{RP}$ & & & 1 \\
\hline Pandion haliaetus & T-MI & & & 1 & collaris & & & & \\
\hline $\begin{array}{l}\text { Chondrohierax } \\
\text { uncinatus }\end{array}$ & $\mathrm{RP}$ & & $\operatorname{Pr}$ & 1 & $\begin{array}{l}\text { Charadrius } \\
\text { wilsonia }\end{array}$ & MI & & & 1 \\
\hline Elanus leucurus & $\mathrm{RP}$ & & & 1 & Charadrius & MI & & & 1 \\
\hline Harpagus & $\mathrm{RP}$ & & $\operatorname{Pr}$ & 1 & semipalmatus & & & & \\
\hline bidentatus & & & & & Charadrius & $\mathrm{RP}$ & & & 1 \\
\hline Circus cyaneus & MI & & & 1 & vociferus & & & & \\
\hline Accipiter striatus & MI & & $\operatorname{Pr}$ & 1 & Haematopus & $\mathrm{RP}$ & & & 1 \\
\hline Accipiter cooperii & MI & & $\operatorname{Pr}$ & 1 & palliatus & & & & \\
\hline $\begin{array}{l}\text { Geranospiza } \\
\quad \text { caerulescens }\end{array}$ & $\mathrm{RP}$ & & A & 1 & $\begin{array}{l}\text { Himantopus } \\
\text { mexicanus }\end{array}$ & $\mathrm{RP}$ & & & 1 \\
\hline Buteo albonotatus & MI & & $\operatorname{Pr}$ & 1 & Recurvirostra & MI & & & 1 \\
\hline Buteo nitidus & $\mathrm{RP}$ & & & 1 & americana & & & & \\
\hline Buteogallus & $\mathrm{RP}$ & & $\operatorname{Pr}$ & 1 & Jacana spinosa & $\mathrm{RP}$ & & & 1 \\
\hline anthracinus & & & & & Tringa & MI & & & 1 \\
\hline Buteogallus & $\mathrm{RP}$ & & $\operatorname{Pr}$ & 1 & melanoleuca & & & & \\
\hline urubitinga & & & & & Tringa flavipes & MI & & & 1 \\
\hline Rupornis & $\mathrm{RP}$ & & & 1 & Tringa solitaria & MI & & & 1 \\
\hline magnirostris & & & & & Tringa & MI & & & 1 \\
\hline Buteo platypterus & MI & & $\operatorname{Pr}$ & 1 & semipalmata & & & & \\
\hline Buteo brachyurus & $\mathrm{RP}$ & & & 1 & Tringa incana & MI & & & 1 \\
\hline Buteo swainsoni & $\mathrm{T}$ & & $\operatorname{Pr}$ & 1 & Actitis macularius & MI & & & 1 \\
\hline $\begin{array}{l}\text { Geranoaetus } \\
\text { albicaudatus }\end{array}$ & $\mathrm{RP}$ & & $\operatorname{Pr}$ & 1 & $\begin{array}{l}\text { Numenius } \\
\text { phaeopus }\end{array}$ & MI & & & 1 \\
\hline Buteo jamaicensis & $\mathrm{RP}$ & & & 1 & Numenius & MI & & & 1 \\
\hline Micrastur & $\mathrm{RP}$ & & $\operatorname{Pr}$ & 1 & americanus & & & & \\
\hline ruficollis & & & & & Arenaria interpres & MI & & & 1 \\
\hline Micrastur & $\mathrm{RP}$ & & $\operatorname{Pr}$ & 1 & Calidris alpina & MI & & & 1 \\
\hline semitorquatus & & & & & Calidris alba & MI & & & 1 \\
\hline Caracara & $\mathrm{RP}$ & & & 1 & Calidris mauri & MI & & & 1 \\
\hline cheriway & & & & & Calidris minutilla & MI & & & 1 \\
\hline Herpetotheres & $\mathrm{RP}$ & & & 1 & Calidris bairdii & $\mathrm{T}$ & & & 1 \\
\hline cachinnans & & & & & Limnodromus & MI & & & 1 \\
\hline Falco sparverius & MI & & & 1 & griseus & & & & \\
\hline Falco columbarius & MI & & & 1 & Limnodromus & MI & & & 1 \\
\hline Falco peregrinus & MI & & $\operatorname{Pr}$ & 1 & scolopaceus & & & & \\
\hline Ortalis & $\mathrm{RP}$ & $\mathrm{E}$ & & 1 & Gallinago delicata & MI & & & 1 \\
\hline poliocephala & & & & & Gallinago & MI & & & 1 \\
\hline Philortyx fasciatus & $\mathrm{RP}$ & $\mathrm{E}$ & & 1 & gallinago & & & & \\
\hline Colinus coyolcos & $\mathrm{RP}$ & $\mathrm{E}$ & & 2 & Phalaropus & $\mathrm{T}$ & & & 1 \\
\hline Cyrtonyx sallei & $\mathrm{RP}$ & & $\operatorname{Pr}$ & 2 & tricolor & & & & \\
\hline Laterallus ruber & $\mathrm{RP}$ & & & 1 & $\begin{array}{l}\text { Phalaropus } \\
\text { lobatus }\end{array}$ & MI & & & 1 \\
\hline
\end{tabular}




\begin{tabular}{|c|c|c|c|c|c|c|c|c|c|}
\hline $\begin{array}{l}\text { Nombre } \\
\text { científico }\end{array}$ & $\begin{array}{l}\text { Estacio- } \\
\text { nalidad }\end{array}$ & Endemismo & $\begin{array}{l}\text { Categoría } \\
\text { de riesgo }\end{array}$ & $\begin{array}{l}\text { Grupo de } \\
\text { acuerdo } \\
\text { con el } \\
\text { número de } \\
\text { registros } \\
\text { obtenidos } \\
\text { para los } \\
\text { MDE }\end{array}$ & $\begin{array}{l}\text { Nombre } \\
\text { científico }\end{array}$ & $\begin{array}{l}\text { Estacio- } \\
\text { nalidad }\end{array}$ & Endemismo & $\begin{array}{l}\text { Categoría } \\
\text { de riesgo }\end{array}$ & $\begin{array}{l}\text { Grupo de } \\
\text { acuerdo } \\
\text { con el } \\
\text { número de } \\
\text { registros } \\
\text { obtenidos } \\
\text { para los } \\
\text { MDE }\end{array}$ \\
\hline $\begin{array}{l}\text { Thalasseus } \\
\text { sandvicensis }\end{array}$ & MI & & & 1 & $\begin{array}{l}\text { Megascops } \\
\text { seductus }\end{array}$ & $\mathrm{RP}$ & $\mathrm{E}$ & A & 2 \\
\hline Leucophaeus & MI & & & 1 & Bubo virginianus & $\mathrm{RP}$ & & & 1 \\
\hline atricilla & & & & & Glaucidium & $\mathrm{RP}$ & $\mathrm{E}$ & A & 1 \\
\hline $\begin{array}{l}\text { Larus } \\
\quad \text { delawarensis }\end{array}$ & MI & & & 1 & $\begin{array}{c}\text { palmarum } \\
\text { Glaucidium }\end{array}$ & $\mathrm{RP}$ & & & 1 \\
\hline Larus argentatus & MI & & & 1 & brasilianum & & & & \\
\hline Leucophaeus & $\mathrm{T}$ & & & 1 & Ciccaba virgata & $\mathrm{RP}$ & & & 1 \\
\hline pipixcan & & & & & Chordeiles & $\mathrm{RP}$ & & & 1 \\
\hline Larus heermanni & $\mathrm{V}$ & & $\operatorname{Pr}$ & 1 & acutipennis & & & & \\
\hline Gelochelidon & M.I & & & 1 & Chordeiles minor & MV & & & 1 \\
\hline nilotica & & & & & Nyctidromus & $\mathrm{RP}$ & & & 1 \\
\hline Hydroprogne & M.I & & & 1 & albicollis & & & & \\
\hline caspia & & & & & Cypseloides niger & MV & & & 1 \\
\hline $\begin{array}{l}\text { Thalasseus } \\
\text { maximus }\end{array}$ & MI & & & 1 & $\begin{array}{l}\text { Streptoprocne } \\
\text { zonaris }\end{array}$ & $\mathrm{RP}$ & & & 1 \\
\hline Thalasseus & V & & $\operatorname{Pr}$ & 1 & Chaetura vauxi & $\mathrm{RP}$ & & & 1 \\
\hline elegans & & & & & Phaethornis & $\mathrm{RP}$ & & & 1 \\
\hline Sterna forsteri & MI & & & 1 & mexicanus & & & & \\
\hline Sterna hirundo & $\mathrm{V}$ & & & 1 & Chlorostilbon & $\mathrm{RP}$ & $\mathrm{E}$ & & 1 \\
\hline Sternula & $\mathrm{RP}$ & & $\operatorname{Pr}$ & 1 & auriceps & & & & \\
\hline antillarum & & & & & Cynanthus & $\mathrm{RP}$ & $\mathrm{E}$ & & 1 \\
\hline Onychoprion & MI & & $\operatorname{Pr}$ & 2 & sordidus & & & & \\
\hline anaethetus & & & & & Cynanthus & $\mathrm{RP}$ & $\mathrm{E}$ & & 1 \\
\hline Chlidonias niger & T-MI & & & 1 & doubledayi & & & & \\
\hline Anous stolidus & MI & & & 2 & Hylocharis & $\mathrm{RP}$ & & & 1 \\
\hline Rynchops niger & MI & & & 1 & leucotis & & & & \\
\hline Patagioenas & $\mathrm{RP}$ & & & 1 & Amazilia beryllina & $\mathrm{RP}$ & & & 1 \\
\hline flavirostris & & & & & Amazilia rutila & $\mathrm{RP}$ & & & 1 \\
\hline Zenaida asiatica & $\mathrm{RP}$ & & & 1 & Amazilia violiceps & $\mathrm{RP}$ & & & 1 \\
\hline Zenaida macroura & $\mathrm{RP}$ & & & 1 & Amazilia & $\mathrm{RP}$ & $\mathrm{E}$ & A & 1 \\
\hline Columbina inca & $\mathrm{RP}$ & & & 1 & viridifrons & & & & \\
\hline $\begin{array}{l}\text { Columbina } \\
\text { passerina }\end{array}$ & $\mathrm{RP}$ & & & 1 & $\begin{array}{l}\text { Lampornis } \\
\quad \text { margaritae }\end{array}$ & $\mathrm{RP}$ & & & 1 \\
\hline Columbina minuta & $\mathrm{RP}$ & & & 1 & Heliomaster & $\mathrm{RP}$ & & $\operatorname{Pr}$ & 1 \\
\hline Columbina & $\mathrm{RP}$ & & & 1 & longirostris & & & & \\
\hline talpacoti & & & & & Heliomaster & $\mathrm{RP}$ & & & 1 \\
\hline Leptotila & $\mathrm{RP}$ & & & 1 & constantii & & & & \\
\hline verreauxi & & & & & Archilochus & T-MI & & & 1 \\
\hline Geotrygon & $\mathrm{RP}$ & & & 1 & colubris & & & & \\
\hline montana & & & & & Archilochus & MI & & & 1 \\
\hline Eupsittula & $\mathrm{RP}$ & & $\operatorname{Pr}$ & 1 & alexandri & & & & \\
\hline canicularis & & & & & Selasphorus & MI & & & 1 \\
\hline Brotogeris & $\mathrm{RP}$ & & A & 1 & calliope & & & & \\
\hline jugularis & & & & & Selasphorus & MI & & & 1 \\
\hline Amazona albifrons & $\mathrm{RP}$ & & $\operatorname{Pr}$ & 1 & platycercus & & & & \\
\hline Amazona finschi & $\mathrm{RP}$ & $\mathrm{E}$ & $\mathrm{P}$ & 1 & Selasphorus rufus & MI & & & 1 \\
\hline Amazona oratrix & $\mathrm{RP}$ & & $\mathrm{P}$ & 1 & Trogon citreolus & $\mathrm{RP}$ & $\mathrm{E}$ & & 1 \\
\hline Coccyzus eryth- & $\mathrm{T}$ & & & 2 & Trogon mexicanus & $\mathrm{RP}$ & & & 1 \\
\hline ropthalmus & & & & & Trogon ambiguus & $\mathrm{RP}$ & & & 1 \\
\hline Coccyzus minor & $\mathrm{RP}$ & & & 1 & Momotus & $\mathrm{RP}$ & & & 1 \\
\hline Piaya mexicana & $\mathrm{RP}$ & $\mathrm{E}$ & & 1 & mexicanus & & & & \\
\hline $\begin{array}{l}\text { Morococcyx } \\
\text { erythropygus }\end{array}$ & $\mathrm{RP}$ & & & 1 & $\begin{array}{c}\text { Megaceryle } \\
\text { torquata }\end{array}$ & $\mathrm{RP}$ & & & 1 \\
\hline Geococcyx velox & $\mathrm{RP}$ & & & 1 & Megaceryle & MI & & & 1 \\
\hline $\begin{array}{l}\text { Crotophaga } \\
\text { sulcirostris }\end{array}$ & $\mathrm{RP}$ & & & 1 & alcyon & & & & \\
\hline
\end{tabular}




\begin{tabular}{|c|c|c|c|c|c|c|c|c|c|}
\hline $\begin{array}{l}\text { Nombre } \\
\text { científico }\end{array}$ & $\begin{array}{l}\text { Estacio- } \\
\text { nalidad }\end{array}$ & Endemismo & $\begin{array}{l}\text { Categoría } \\
\text { de riesgo }\end{array}$ & $\begin{array}{l}\text { Grupo de } \\
\text { acuerdo } \\
\text { con el } \\
\text { número de } \\
\text { registros } \\
\text { obtenidos } \\
\text { para los } \\
\text { MDE }\end{array}$ & $\begin{array}{l}\text { Nombre } \\
\text { científico }\end{array}$ & $\begin{array}{l}\text { Estacio- } \\
\text { nalidad }\end{array}$ & Endemismo & $\begin{array}{l}\text { Categoría } \\
\text { de riesgo }\end{array}$ & $\begin{array}{l}\text { Grupo de } \\
\text { acuerdo } \\
\text { con el } \\
\text { número de } \\
\text { registros } \\
\text { obtenidos } \\
\text { para los } \\
\text { MDE }\end{array}$ \\
\hline $\begin{array}{c}\text { Chloroceryle } \\
\text { amazona }\end{array}$ & $\mathrm{RP}$ & & & 1 & $\begin{array}{l}\text { Myiarchus } \\
\quad \text { cinerascens }\end{array}$ & MI & & & 1 \\
\hline $\begin{array}{c}\text { Chloroceryle } \\
\text { americana }\end{array}$ & $\mathrm{RP}$ & & & 1 & $\begin{array}{r}\text { Myiarchus } \\
\text { nuttingi }\end{array}$ & $\mathrm{RP}$ & & & 1 \\
\hline $\begin{array}{l}\text { Aulacorhynchus } \\
\quad \text { wagleri }\end{array}$ & $\mathrm{RP}$ & & $\operatorname{Pr}$ & 1 & $\begin{array}{l}\text { Myiarchus } \\
\text { tyrannulus }\end{array}$ & $\mathrm{RP}$ & & & 1 \\
\hline $\begin{array}{l}\text { Melanerpes } \\
\text { formicivorus }\end{array}$ & $\mathrm{RP}$ & & & 1 & $\begin{array}{r}\text { Deltarhynchus } \\
\text { flammulatus }\end{array}$ & $\mathrm{RP}$ & $\mathrm{E}$ & $\operatorname{Pr}$ & 1 \\
\hline $\begin{array}{l}\text { Melanerpes } \\
\text { chrysogenys }\end{array}$ & $\mathrm{RP}$ & $\mathrm{E}$ & & 1 & $\begin{array}{l}\text { Pitangus } \\
\text { sulphuratus }\end{array}$ & $\mathrm{RP}$ & & & 1 \\
\hline $\begin{array}{l}\text { Sphyrapicus } \\
\text { varius }\end{array}$ & MI & & & 1 & $\begin{array}{l}\text { Megarynchus } \\
\text { pitangua }\end{array}$ & $\mathrm{RP}$ & & & 1 \\
\hline Picoides scalaris & $\mathrm{RP}$ & & & 1 & Myiozetetes & $\mathrm{RP}$ & & & 1 \\
\hline Colaptes & $\mathrm{RP}$ & $\mathrm{E}$ & & 1 & similis & & & & \\
\hline $\begin{array}{l}\text { auricularis } \\
\text { Dryocopus }\end{array}$ & $\mathrm{RP}$ & & & 1 & $\begin{array}{r}\text { Myiodynastes } \\
\text { luteiventris }\end{array}$ & MV & & & 1 \\
\hline lineatus & & & & & Tyrannus & $\mathrm{RP}$ & & & 1 \\
\hline $\begin{array}{l}\text { Campephilus } \\
\text { guatemalensis }\end{array}$ & $\mathrm{RP}$ & & $\operatorname{Pr}$ & 1 & $\begin{array}{l}\text { melancholicus } \\
\text { Tyrannus }\end{array}$ & $\mathrm{RP}$ & & & 1 \\
\hline $\begin{array}{l}\text { Sittasomus } \\
\quad \text { griseicapillus }\end{array}$ & $\mathrm{RP}$ & & & 1 & $\begin{array}{l}\text { vociferans } \\
\text { Tyrannus }\end{array}$ & $\mathrm{RP}$ & $\mathrm{E}$ & & 1 \\
\hline $\begin{array}{l}\text { Xiphorhynchus } \\
\text { flavigaster }\end{array}$ & $\mathrm{RP}$ & & & 1 & $\begin{array}{l}\text { crassirostris } \\
\text { Tyrannus }\end{array}$ & MI & & & 1 \\
\hline Lepidocolaptes & $\mathrm{RP}$ & $\mathrm{E}$ & & 1 & verticalis & & & & \\
\hline leucogaster & & & & & Tyrannus & T-MI & & & 1 \\
\hline Lepidocolaptes & $\mathrm{RP}$ & & & 1 & forficatus & & & & \\
\hline $\begin{array}{c}\text { souleyetii } \\
\text { Camptostoma }\end{array}$ & $\mathrm{RP}$ & & & 1 & $\begin{array}{c}\text { Pachyramphus } \\
\text { uropygialis }\end{array}$ & $\mathrm{RP}$ & $\mathrm{E}$ & & 1 \\
\hline imberbe & & & & & Pachyramphus & $\mathrm{RP}$ & $\mathrm{E}$ & & 1 \\
\hline $\begin{array}{l}\text { Myiopagis } \\
\text { viridicata }\end{array}$ & $\mathrm{RP}$ & & & 1 & $\begin{array}{c}\text { albiventris } \\
\text { Tityra griseiceps }\end{array}$ & $\mathrm{RP}$ & $\mathrm{E}$ & & 1 \\
\hline $\begin{array}{l}\text { Xenotriccus } \\
\quad \text { mexicanus }\end{array}$ & $\mathrm{RP}$ & $\mathrm{E}$ & $\operatorname{Pr}$ & 1 & $\begin{array}{l}\text { Lanius } \\
\quad \text { ludovicianus }\end{array}$ & $\mathrm{RP}$ & & & 1 \\
\hline Contopus cooperi & T-MI & & & 1 & Vireo bellii & T-MI & & & 1 \\
\hline Contopus pertinax & $\mathrm{RP}$ & & & 1 & Vireo cassinii & MI & & & 1 \\
\hline Contopus & $\mathrm{T}$ & & & 1 & Vireo plumbeus & $\mathrm{RP}$ & & & 1 \\
\hline sordidulus & & & & & Vireo huttoni & $\mathrm{RP}$ & & & 1 \\
\hline Empidonax traillii & MI & & & 1 & Vireo & $\mathrm{RP}$ & $\mathrm{E}$ & & 1 \\
\hline Empidonax & MI & & & 1 & hypochryseus & & & & \\
\hline albigularis & & & & & Vireo pallens & $\mathrm{RP}$ & E & $\operatorname{Pr}$ & 1 \\
\hline Empidonax & MI & & & 1 & Vireo swainsonii & $\mathrm{RP}$ & & & 1 \\
\hline minimus & & & & & Vireo & $\mathrm{V}$ & & & 1 \\
\hline Empidonax & T-MI & & & 1 & philadelphicus & & & & \\
\hline hammondii & & & & & Vireo flavoviridis & MV & & & 1 \\
\hline $\begin{array}{l}\text { Empidonax } \\
\text { oberholseri }\end{array}$ & MI & & & 1 & $\begin{array}{l}\text { Vireolanius } \\
\quad \text { melitophrys }\end{array}$ & $\mathrm{RP}$ & & & 1 \\
\hline Empidonax & MI & & & 1 & Calocitta formosa & $\mathrm{RP}$ & & & 1 \\
\hline difficilis & & & & & Cyanocorax & $\mathrm{RP}$ & $\mathrm{E}$ & & 1 \\
\hline Empidonax & $\mathrm{RP}$ & & & 1 & speciosa & & & & \\
\hline occidentalis & & & & & Cyanocorax & $\mathrm{RP}$ & $\mathrm{E}$ & & 1 \\
\hline Sayornis nigricans & $\mathrm{RP}$ & & & 1 & sanblasianus & & & & \\
\hline $\begin{array}{l}\text { Pyrocephalus } \\
\text { rubinus }\end{array}$ & $\mathrm{RP}$ & & & 1 & $\begin{array}{l}\text { Aphelocoma } \\
\text { sumichrasti }\end{array}$ & $\mathrm{RP}$ & $\mathrm{E}$ & & 1 \\
\hline Attila pacificus & $\mathrm{RP}$ & $\mathrm{E}$ & & 1 & Corvus sinuatus & $\mathrm{RP}$ & & & 1 \\
\hline Myiarchus & $\mathrm{RP}$ & & & 1 & Progne subis & $\mathrm{T}$ & & & 1 \\
\hline tuberculifer & & & & & $\begin{array}{l}\text { Tachycineta } \\
\text { bicolor }\end{array}$ & MI & & & 1 \\
\hline
\end{tabular}




\begin{tabular}{|c|c|c|c|c|c|c|c|c|c|}
\hline $\begin{array}{l}\text { Nombre } \\
\text { científico }\end{array}$ & $\begin{array}{l}\text { Estacio- } \\
\text { nalidad }\end{array}$ & Endemismo & $\begin{array}{l}\text { Categoría } \\
\text { de riesgo }\end{array}$ & $\begin{array}{l}\text { Grupo de } \\
\text { acuerdo } \\
\text { con el } \\
\text { número de } \\
\text { registros } \\
\text { obtenidos } \\
\text { para los } \\
\text { MDE }\end{array}$ & $\begin{array}{l}\text { Nombre } \\
\text { científico }\end{array}$ & $\begin{array}{l}\text { Estacio- } \\
\text { nalidad }\end{array}$ & Endemismo & $\begin{array}{l}\text { Categoría } \\
\text { de riesgo }\end{array}$ & $\begin{array}{l}\text { Grupo de } \\
\text { acuerdo } \\
\text { con el } \\
\text { número de } \\
\text { registros } \\
\text { obtenidos } \\
\text { para los } \\
\text { MDE }\end{array}$ \\
\hline Progne chalybea & RP & & & 1 & Oreothlypis & MI & & & 1 \\
\hline $\begin{array}{c}\text { Tachycineta } \\
\text { albilinea }\end{array}$ & $\mathrm{RP}$ & & & 1 & $\begin{array}{l}\text { peregrina } \\
\text { Oreothlypis celata }\end{array}$ & MI & & & 1 \\
\hline $\begin{array}{l}\text { Tachycineta } \\
\text { thalassina }\end{array}$ & MI & & & 1 & $\begin{array}{l}\text { Oreothlypis } \\
\quad \text { ruficapilla }\end{array}$ & MI & & & 1 \\
\hline $\begin{array}{l}\text { Stelgidopteryx } \\
\text { serripennis }\end{array}$ & $\mathrm{RP}$ & & & 1 & $\begin{array}{l}\text { Oreothlypis luciae } \\
\text { Setophaga citrina }\end{array}$ & $\begin{array}{l}\text { MI } \\
\text { MI }\end{array}$ & & & $\begin{array}{l}1 \\
1\end{array}$ \\
\hline Riparia riparia & T-MI & & & 1 & Setophaga & MI & & & 1 \\
\hline $\begin{array}{l}\text { Petrochelidon } \\
\text { melanogaster }\end{array}$ & $\mathrm{T}$ & & & 2 & $\begin{array}{l}\text { americana } \\
\text { Setophaga }\end{array}$ & $\mathrm{RP}$ & $\mathrm{E}$ & & 1 \\
\hline Hirundo rustica & MI & & & 1 & nigrilora & & & & \\
\hline $\begin{array}{l}\text { Psaltriparus } \\
\text { melanotis }\end{array}$ & $\mathrm{RP}$ & & & 2 & $\begin{array}{l}\text { Setophaga aestiva } \\
\text { Setophaga }\end{array}$ & $\begin{array}{l}\text { MI } \\
\text { MI }\end{array}$ & & & $\begin{array}{l}1 \\
1\end{array}$ \\
\hline $\begin{array}{l}\text { Certhia } \\
\quad \text { americana }\end{array}$ & $\mathrm{RP}$ & & & 1 & $\begin{array}{l}\text { magnolia } \\
\text { Setophaga }\end{array}$ & MI & & & 1 \\
\hline $\begin{array}{l}\text { Campylorhynchus } \\
\text { humilus }\end{array}$ & $\mathrm{RP}$ & $\mathrm{E}$ & & 2 & $\begin{array}{l}\text { auduboni } \\
\text { Setophaga virens }\end{array}$ & MI & & & 1 \\
\hline $\begin{array}{l}\text { Campylorhynchus } \\
\text { gularis }\end{array}$ & $\mathrm{RP}$ & $\mathrm{E}$ & & 1 & $\begin{array}{l}\text { Setophaga } \\
\quad \text { dominica }\end{array}$ & MI & & & 1 \\
\hline $\begin{array}{l}\text { Catherpes } \\
\quad \text { mexicanus }\end{array}$ & $\mathrm{RP}$ & & & 1 & $\begin{array}{l}\text { Setophaga graciae } \\
\text { Setophaga }\end{array}$ & $\begin{array}{l}\text { RP } \\
\text { T-MI }\end{array}$ & & & $\begin{array}{l}1 \\
1\end{array}$ \\
\hline $\begin{array}{l}\text { Thryophilus } \\
\text { sinaloa }\end{array}$ & $\mathrm{RP}$ & $\mathrm{E}$ & & 1 & $\begin{array}{l}\text { ruticilla } \\
\text { Mniotilta varia }\end{array}$ & MI & & & 1 \\
\hline $\begin{array}{l}\text { Thryophilus } \\
\quad \text { pleurostictus }\end{array}$ & $\mathrm{RP}$ & & & 1 & $\begin{array}{l}\text { Seiurus } \\
\quad \text { aurocapilla }\end{array}$ & MI & & & 1 \\
\hline $\begin{array}{l}\text { Pheugopedius } \\
\text { felix }\end{array}$ & $\mathrm{RP}$ & $\mathrm{E}$ & & 1 & $\begin{array}{l}\text { Parkesia } \\
\quad \text { noveboracensis }\end{array}$ & MI & & & 1 \\
\hline Thryomanes & $\mathrm{RP}$ & & & 1 & Parkesia motacilla & MI & & & 1 \\
\hline $\begin{array}{l}\text { bewickii } \\
\text { Troglodytes } \\
\text { brunneicollis }\end{array}$ & MI & & & 1 & $\begin{array}{l}\text { Geothlypis tolmiei } \\
\text { Geothlypis } \\
\quad \text { melanops }\end{array}$ & $\begin{array}{l}\text { MI } \\
\text { MI }\end{array}$ & & A & $\begin{array}{l}1 \\
1\end{array}$ \\
\hline $\begin{array}{l}\text { Henicorhina } \\
\text { leucophrys }\end{array}$ & $\mathrm{RP}$ & & & 1 & $\begin{array}{l}\text { Geothlypis } \\
\quad \text { poliocephala }\end{array}$ & $\mathrm{RP}$ & & & 1 \\
\hline Uropsila pacifica & RP & $\mathrm{E}$ & & 1 & Cardellina pusilla & MI & & & 1 \\
\hline Regulus calendula & MI & & & 1 & Myioborus pictus & $\mathrm{RP}$ & & & 1 \\
\hline $\begin{array}{l}\text { Polioptila } \\
\quad \text { caerulea }\end{array}$ & MI & & & 1 & $\begin{array}{l}\text { Myioborus } \\
\text { miniatus }\end{array}$ & $\mathrm{RP}$ & & & 1 \\
\hline $\begin{array}{l}\text { Polioptila } \\
\text { albiloris }\end{array}$ & $\mathrm{RP}$ & & & 1 & $\begin{array}{l}\text { Basileuterus } \\
\quad \text { lachrymosus }\end{array}$ & $\mathrm{RP}$ & & & 1 \\
\hline Sialia sialis & RP & & & 1 & Basileuterus & RP & & & 1 \\
\hline $\begin{array}{l}\text { Myadestes } \\
\text { occidentalis }\end{array}$ & $\mathrm{RP}$ & & $\operatorname{Pr}$ & 1 & $\begin{array}{c}\text { rufifrons } \\
\text { Basileuterus }\end{array}$ & $\mathrm{RP}$ & & & 1 \\
\hline $\begin{array}{l}\text { Catharus } \\
\quad \text { aurantiirostris }\end{array}$ & $\mathrm{RP}$ & & & 1 & $\begin{array}{l}\text { culicivorus } \\
\text { Icteria virens }\end{array}$ & T-MI & & & 1 \\
\hline $\begin{array}{l}\text { Catharus } \\
\text { ustulatus }\end{array}$ & T-MI & & & 1 & $\begin{array}{l}\text { Granatellus } \\
\text { venustus }\end{array}$ & $\mathrm{RP}$ & $\mathrm{E}$ & & 1 \\
\hline Catharus guttatus & MI & & & 1 & Rhodinocichla & $\mathrm{RP}$ & $\mathrm{E}$ & & 1 \\
\hline Turdus assimilis & $\mathrm{RP}$ & & & 1 & schistacea & & & & \\
\hline $\begin{array}{l}\text { Turdus } \\
\quad \text { rufopalliatus }\end{array}$ & $\mathrm{RP}$ & $\mathrm{E}$ & & 1 & $\begin{array}{l}\text { Habia affinis } \\
\text { Piranga hepatica }\end{array}$ & $\begin{array}{l}\mathrm{RP} \\
\mathrm{RP}\end{array}$ & $\mathrm{E}$ & & $\begin{array}{l}1 \\
1\end{array}$ \\
\hline $\begin{array}{l}\text { Turdus } \\
\quad \text { migratorius }\end{array}$ & $\mathrm{RP}$ & & & 1 & $\begin{array}{l}\text { Piranga rubra } \\
\text { Piranga }\end{array}$ & $\begin{array}{l}\text { MI } \\
\text { MI }\end{array}$ & & & $\begin{array}{l}1 \\
1\end{array}$ \\
\hline Mimus polyglottos & $\mathrm{RP}$ & & & 1 & ludoviciana & & & & \\
\hline $\begin{array}{l}\text { Melanotis } \\
\text { caerulescens }\end{array}$ & $\mathrm{RP}$ & $\mathrm{E}$ & & 1 & $\begin{array}{l}\text { Piranga bidentata } \\
\text { Piranga }\end{array}$ & $\begin{array}{l}\mathrm{RP} \\
\mathrm{RP}\end{array}$ & $\mathrm{E}$ & & $\begin{array}{l}1 \\
1\end{array}$ \\
\hline $\begin{array}{l}\text { Bombycilla } \\
\text { cedrorum }\end{array}$ & MI & & & 1 & $\begin{array}{l}\text { leucoptera } \\
\text { Thraupis } \\
\text { episcopus }\end{array}$ & $\mathrm{RP}$ & & & 1 \\
\hline
\end{tabular}




\begin{tabular}{|c|c|c|c|c|}
\hline $\begin{array}{l}\text { Nombre } \\
\text { científico }\end{array}$ & $\begin{array}{l}\text { Estacio- } \\
\text { nalidad }\end{array}$ & Endemismo & $\begin{array}{l}\text { Categoría } \\
\text { de riesgo }\end{array}$ & $\begin{array}{l}\text { Grupo de } \\
\text { acuerdo } \\
\text { con el } \\
\text { número de } \\
\text { registros } \\
\text { obtenidos } \\
\text { para los } \\
\text { MDE }\end{array}$ \\
\hline $\begin{array}{l}\text { Euphonia } \\
\text { godmani }\end{array}$ & $\mathrm{RP}$ & $\mathrm{E}$ & & 1 \\
\hline $\begin{array}{l}\text { Euphonia } \\
\quad \text { elegantissima }\end{array}$ & $\mathrm{RP}$ & & & 1 \\
\hline $\begin{array}{l}\text { Cyanerpes } \\
\text { cyaneus }\end{array}$ & $\mathrm{RP}$ & & & 1 \\
\hline Volatinia jacarina & RP & & & 1 \\
\hline $\begin{array}{l}\text { Sporophila } \\
\text { torqueola }\end{array}$ & $\mathrm{RP}$ & E & & 1 \\
\hline Sporophila minuta & $\mathrm{RP}$ & & & 1 \\
\hline $\begin{array}{l}\text { Arremonops } \\
\text { sumichrasti }\end{array}$ & $\mathrm{RP}$ & $\mathrm{E}$ & & 2 \\
\hline Melozone fusca & $\mathrm{RP}$ & & & 1 \\
\hline $\begin{array}{l}\text { Peucaea } \\
\quad \text { humeralis }\end{array}$ & $\mathrm{RP}$ & $\mathrm{E}$ & & 1 \\
\hline $\begin{array}{l}\text { Peucaea } \\
\quad \text { acuminata }\end{array}$ & $\mathrm{RP}$ & E & & 2 \\
\hline $\begin{array}{l}\text { Aimophila } \\
\text { rufescens }\end{array}$ & $\mathrm{RP}$ & & & 1 \\
\hline $\begin{array}{l}\text { Chondestes } \\
\text { grammacus }\end{array}$ & MI & & & 1 \\
\hline $\begin{array}{l}\text { Passerculus } \\
\quad \text { sandwichensis }\end{array}$ & MI & & & 1 \\
\hline $\begin{array}{l}\text { Ammodramus } \\
\text { savannarum }\end{array}$ & MI & & & 1 \\
\hline $\begin{array}{l}\text { Melospiza } \\
\quad \text { lincolnii }\end{array}$ & MI & & & 1 \\
\hline $\begin{array}{l}\text { Melospiza } \\
\quad \text { georgiana }\end{array}$ & MI & & & 1 \\
\hline Saltator vigorsii & $\mathrm{RP}$ & $\mathrm{E}$ & & 1 \\
\hline Saltator atriceps & $\mathrm{RP}$ & & & 1 \\
\hline $\begin{array}{c}\text { Cardinalis } \\
\text { carneus }\end{array}$ & $\mathrm{RP}$ & & & 1 \\
\hline $\begin{array}{l}\text { Pheucticus } \\
\quad \text { ludovicianus }\end{array}$ & MI & & & 1 \\
\hline $\begin{array}{l}\text { Pheucticus } \\
\quad \text { chrysopeplus }\end{array}$ & $\mathrm{RP}$ & $\mathrm{E}$ & & 1 \\
\hline $\begin{array}{l}\text { Pheucticus } \\
\quad \text { melanocephalus }\end{array}$ & $\mathrm{RP}$ & & & 1 \\
\hline $\begin{array}{l}\text { Cyanocompsa } \\
\text { indigotica }\end{array}$ & $\mathrm{RP}$ & $\mathrm{E}$ & & 1 \\
\hline $\begin{array}{l}\text { Passerina } \\
\text { caerulea }\end{array}$ & $\mathrm{RP}$ & & & 1 \\
\hline Passerina cyanea & MI & & & 1 \\
\hline $\begin{array}{l}\text { Passerina } \\
\text { versicolor }\end{array}$ & $\mathrm{RP}$ & & & 1 \\
\hline $\begin{array}{l}\text { Passerina } \\
\quad \text { leclancherii }\end{array}$ & $\mathrm{RP}$ & $\mathrm{E}$ & & 1 \\
\hline Passerina ciris & MI & & $\operatorname{Pr}$ & 1 \\
\hline Spiza americana & MI & & & 1 \\
\hline $\begin{array}{l}\text { Agelaius } \\
\quad \text { phoeniceus }\end{array}$ & $\mathrm{RP}$ & & & 1 \\
\hline Sturnella magna & $\mathrm{RP}$ & & & 1 \\
\hline $\begin{array}{l}\text { Xanthocephalus } \\
\text { xanthocephalus }\end{array}$ & MI & & & 1 \\
\hline $\begin{array}{l}\text { Euphagus } \\
\text { cyanocephalus }\end{array}$ & MI & & & 1 \\
\hline
\end{tabular}

\begin{tabular}{|c|c|c|c|c|}
\hline $\begin{array}{l}\text { Nombre } \\
\text { científico }\end{array}$ & $\begin{array}{l}\text { Estacio- } \\
\text { nalidad }\end{array}$ & Endemismo & $\begin{array}{l}\text { Categoría } \\
\text { de riesgo }\end{array}$ & $\begin{array}{l}\text { Grupo de } \\
\text { acuerdo } \\
\text { con el } \\
\text { número de } \\
\text { registros } \\
\text { obtenidos } \\
\text { para los } \\
\text { MDE }\end{array}$ \\
\hline $\begin{array}{l}\text { Quiscalus } \\
\quad \text { mexicanus }\end{array}$ & $\mathrm{RP}$ & & & 1 \\
\hline Molothrus aeneus & RP & & & 1 \\
\hline Molothrus ater & $\mathrm{RP}$ & & & 1 \\
\hline Icterus wagleri & $\mathrm{RP}$ & & & 1 \\
\hline Icterus fuertesi & MI & & & 2 \\
\hline Icterus cucullatus & MI & & & 1 \\
\hline Icterus pustulatus & $\mathrm{RP}$ & $\mathrm{E}$ & & 1 \\
\hline Icterus pectoralis & $\mathrm{RP}$ & & & 1 \\
\hline Icterus gularis & $\mathrm{RP}$ & & & 1 \\
\hline Icterus dickeyae & $\mathrm{RP}$ & $\mathrm{E}$ & & 2 \\
\hline Icterus galbula & MI & & & 1 \\
\hline Icterus bullockii & MI & & & 1 \\
\hline $\begin{array}{l}\text { Cacicus } \\
\quad \text { melanicterus }\end{array}$ & $\mathrm{RP}$ & $\mathrm{E}$ & & 1 \\
\hline $\begin{array}{r}\text { Haemorhous } \\
\text { mexicanus }\end{array}$ & $\mathrm{RP}$ & & & 1 \\
\hline Spinus pinus & MI & & & 1 \\
\hline Spinus psaltria & $\mathrm{RP}$ & & & 1 \\
\hline
\end{tabular}

\section{Referencias}

Almazán-Núñez, R. C., Almazán-Juárez, A. y Ruiz-Gutiérrez, F. (2011). Áreas comunitarias para la conservación de los recursos biológicos de la Sierra Madre del Sur, Guerrero, México. Universidad y Ciencia, 27, 315-329.

Almazán-Núñez, R. C., López-de Aquino, S., Ríos-Muñoz, C. A. y NavarroSigüenza, A. G. (2013). Áreas potenciales de riqueza, endemismo y conservación de las aves del estado de Querétaro, México. Interciencia, $38,26-34$.

Almazán-Núñez, R. C., Meléndez-Herrada, A., García-Vega, C. S., SierraMorales, P. y Méndez-Bahena, A. (2017). Hooded warbler (Setophaga citrina) and other noteworthy bird records from Guerrero, Mexico. Huitzil, $18,16-23$.

Almazán-Núñez, R. C. y Nova-Muñoz, O. (2006). La guacamaya verde (Ara militaris) en la Sierra Madre del Sur, Guerrero, México. Huitzil, 7, 20-22.

Anderson, R. P., Lew, D. y Peterson, A. T. (2003). Evaluating predictive models of species' distributions: criteria for selecting optimal models. Ecological Modelling, 162, 211-232.

Arizmendi, M. C. y Márquez-Valdelamar, L. (2000). Áreas de importancia para la conservación de las aves de México. México, D.F.: Conabio.

A.O.U. (American Ornithologists' Union). (1998). Checklist of North American birds. Washington, D.C.: American Ornithologists' Union.

Bélisle, M. (2005). Measuring landscape connectivity: the challenge of behavioral landscape ecology. Ecology, 86, 1988-1995.

Bezaury-Creel, J. E., Torres, J. F., Ochoa-Ochoa, L. M. y Castro-Campos, M. (2012). Áreas naturales protegidas y otros espacios dedicados a la conservación, restauración y uso sustentable de la biodiversidad en México. In Capas ArcGis [CD-ROM]. México, D.F.: The Nature Conservancy.

Botello, F., Sánchez-Cordero, V. y Ortega-Huerta, M. A. (2015). Disponibilidad de hábitats adecuados para especies de mamíferos a escalas regional (estado de Guerrero) y nacional (México). Revista Mexicana de Biodiversidad, 86, 226-237.

Carreto-Pérez, B. E., Almazán-Juárez, Á., Sierra-Morales, P. y Almazán-Núñez, R. C. (2015). Estudio florístico de la cuenca baja del río Papagayo, Guerrero, México. Polibotánica, 40, 1-27. 
Chazdon, R. L., Colwell, R. K., Denslow, J. S. y Guariguata, M. R. (1998). Statistical methods for estimating species richness of woody regeneration in primary and secondary rain forest of northeastern Costa Rica. En F. Dallmeier y J. A. Comiskey (Eds.), Forest biodiversity research, monitoring and modelling (pp. 285-309). París: The Parthenon Publishing Group.

Colwell, R.K. (2006). EstimateS: statistical estimation of species richness and shared species from samples. Version 9 [consultado 08 Ene 2016]. Disponible en: http://viceroy.eeb.uconn.edu/estimates

Colwell, R. K. y Coddington, J. A. (1994). Estimating terrestrial biodiversity through extrapolation. Philosophical Transactions of the Royal Society of London B: Biological Sciences, 345, 101-118.

Cuervo-Robayo, A. P. y Monroy-Vilchis, O. (2012). Distribución potencial del jaguar Panthera onca (Carnivora: Felidae) en Guerrero México: persistencia de zonas para su conservación. Revista de Biologia Tropical, 60, 1357-1367.

Cuervo-Robayo, A. P., Téllez-Valdés, O., Gómez, M., Venegas-Barrera, C., Manjarrez, J. y Martínez-Meyer, E. (2013). An update of high-resolution monthly climate surfaces for Mexico. International Journal of Climatology, 34, 2427-2437.

Davis, W. B. (1944). Notes on summer birds of Guerrero. The Condor, 46, 9-14.

Diario Oficial de la Federación (DOF). (2010). Decreto por el que se declara al predio «Bicentenario» como Área Natural Protegida bajo la modalidad de Parque Estatal. Chilpancingo, Guerrero, México: Diario Oficial de la Federación., 12 de noviembre del 2010.

Diario Oficial de la Federación (DOF). (2010). Decreto por el que se declara al predio «El Limón» como Área Natural Protegida bajo la modalidad de Parque Estatal. Chilpancingo, Guerrero, México: Diario Oficial de la Federación., 16 de diciembre del 2011.

Diario Oficial de la Federación (DOF). (2012). Decreto por el que se declara al predio «El Limón» como Área Natural Protegida bajo la modalidad de Parque Estatal. Chilpancingo, Guerrero, México: Diario Oficial de la Federación., 16 de febrero del 2012.

Dirzo, R., Young, H. S., Mooney, H. A. y Ceballos, G. (2011). Seasonally dry tropical forests: ecology and conservation. Washington, D.C.: Island Press.

Escalante, T. (2009). Un ensayo sobre regionalización biogeográfica. Revista Mexicana de Biodiversidad, 80, 551-560.

Escalante, T. (2011). De cómo el análisis de parsimonia de endemismos (PAE) tampoco explica la selección natural. Revista Mexicana de Biodiversidad, $82,1057-1059$.

Escalante, T. y Morrone, J. J. (2003). ¿Para qué sirve el análisis de parsimonia de endemismos? En J. J. Morrone y J. Llorente-Bousquets (Eds.), Una perspectiva latinoamericana de la biogeografía (pp. 167-172). México, D.F.: Conabio-UNAM.

Escalante, P., Navarro, A. G. y Peterson, A. T. (1998). Un análisis geográfico ecológico e histórico de la diversidad de aves terrestres en México. En T. P. Ramamoorthy, R. Bye, A. Lot, y J. Fa (Eds.), Diversidad biológica de México: orígenes y distribución (pp. 279-304). México, D.F.: Instituto de Biología, Universidad Nacional Autónoma de México.

Escalante, T., Sánchez-Cordero, V., Morrone, J. J. y Linaje, M. (2007). Areas of endemism of Mexican terrestrial mammals: a case study using species' ecological niche modeling, parsimony analysis of endemicity and Goloboff fit. Interciencia, 32, 151-159.

Esselman, P. C. y Allan, J. D. (2011). Application of species distribution models and conservation planning software to the design of a reserve network for the riverine fishes of northeastern Mesoamerica. Freshwater Biology, 56, $71-88$.

Feria-Arroyo, T. P. y Peterson, A. T. (2002). Prediction of bird community composition based on point-occurrence data and inferential algorithms: a valuable tool in biodiversity assessments. Diversity and Distributions, 8 , $49-56$.

García-Trejo, E. A. y Navarro-Sigüenza, A. G. (2004). Patrones biogeográficos de la riqueza de especies y el endemismo de la avifauna en el oeste de México. Acta Zoológica Mexicana, 20, 167-185.

Gaviño-de la Torre, G., Martínez, A. G., Uribe, Z. P. y Santillán, S. A. (1979). Vertebrados terrestres y vegetación dominante en isla Ixtapa, Guerrero. Anales del Instituto de Biología, Universidad Nacional Autónoma de México. Serie Zoología, 50, 701-719.

Gil, G. y Moreno, C. E. (2007). Los análisis de complementariedad aplicados a la selección de reservas de la biosfera: efecto de la escala. En G.
Halffter, S. Guevara, y A. Melic (Eds.), Hacia una cultura de conservación de la diversidad biológica (pp. 63-70). Zaragoza: Sociedad Entomológica Aragonesa.

Goloboff, P.A., Farris, J.S. y Nixon, K. (2000). TNT: tree analysis using new technology [consultado 15 Oct 2015]. Disponible en: http://www. cladistics.com.

Gómez-de Silva, H. G. y Alvarado, R. E. (2010). Breve historia de la observación de aves en México en el siglo xx y principios del siglo xxI. Huitzil, 11, 9-20.

Gómez-de Silva, H. G. y Medellín, R. A. (2001). Evaluating completeness of species lists for conservation and macroecology: a case study of Mexican land birds. Conservation Biology, 15, 1384-1395.

Gotelli, N. J. y Colwell, R. K. (2011). Estimating species richness. En A. E. Magurran y B. J. McGill (Eds.), Frontiers in measurement biodiversity (pp. 39-54). New York: Oxford University Press.

Greene, E., Wilcove, D. y Mcfarland, M. (1984). Observations of birds at an army ant swarm in Guerrero, Mexico. Condor, 86, 192-193.

Guisan, A., Lehmann, A., Ferrier, S., Austin, M., Overton, J., Aspinall, R., et al. (2006). Making better biogeographical predictions of species' distributions. Journal of Applied Ecology, 43, 386-392.

Guisan, A. y Zimmermann, N. E. (2000). Predictive habitat distribution models in ecology. Ecological Modelling, 135, 147-186.

Haig, S., Mehlman, D. y Oring, L. W. (1998). Avian movements and wetland connectivity in landscape conservation. Conservation Biology, 12, 749-758.

Hortal, J., Borges, P. A. y Gaspar, C. (2006). Evaluating the performance of species richness estimators: sensitivity to sample grain size. Journal of Animal Ecology, 75, 274-287.

Howell, S. N. G. y Webb, S. (1995). A guide to the birds of México and Northem Central America. New York: Oxford University Press.

Humphries, C. J., van Wright, R. I. y Williams, P. H. (1991). Biodiversity reserves: setting new priorities for the conservation of wildlife. Parks, 2, 34-38.

Hutto, R. L. (1995). Can patterns of vegetation change in western Mexico explain population trends in western Neotropical migrants? En M. Wilson y S. Sader (Eds.), Conservation of neotropical migratory birds in Mexico (pp. 48-58). Orono, Maine: Maine Agricultural and Forest Experiment Station, Miscellaneous Publicaion, 727

Illoldi-Rangel, P., Fuller, T., Linaje, M., Pappas, C., Sánchez-Cordero, V. y Sarkar, S. (2008). Solving the maximum representation problem to prioritize areas for the conservation of terrestrial mammals at risk in Oaxaca. Diversity and Distributions, 14, 493-508.

Koleff, P. y Moreno, E. (2006). Áreas protegidas de México y representación de la riqueza. En J. Llorente-Bousquets y J. J. Morrone (Eds.), Regionalización biogeográfica en Iberoamérica y tópicos afines (pp. 351-373). México, D.F.: CYTED-UNAM-Conabio.

Koleff, P., Tambutti, M., March, I. J., Esquivel, R., Cantú, C. y Lira-Noriega, A. (2009). Identificación de prioridades y análisis de vacíos y omisiones en la conservación de la biodiversidad de México En Capital natural de México, Vol. II: Estado de conservación y tendencias de cambio (pp. 651718). México, D.F.: Conabio.

Koleff, P. y Urquiza-Haas, T. (2011). Conservación de la biodiversidad de México: planeación prioridades y perspectivas. En P. Koleff y T. UrquizaHaas (Eds.), Planeación para la conservación de la biodiversidad terrestre en México: retos en un país megadiverso (pp. 11-20). México, D.F.: Conabio-Conanp.

Komar, O. (2003). En las listas faunísticas no todas las especies son iguales: las aves de El Salvador como un ejemplo. En H. Gómez de Silva y A. Oliveras de Ita (Eds.), Conservación de aves: experiencias en México (pp. 57-61). México, D.F.: Cipamex-Conabio.

Lara-Lara, J. R., Arreola-Lizárraga, J. A., Calderón-Aguilera, L. E., CamachoIbar, V. F., De la Lanza-Espino, G., Escofet-Giansone, A., et al. (2008). . pp. 109-134. Los ecosistemas costeros, insulares y epicontinentales. En Capital natural de México (Vol. 1) México, D.F: Conabio. Conocimiento actual de la biodiversidad.

Loiselle, B. A., Howell, C. A., Graham, C. H., Goerck, J. M., Brooks, T., Smith, K. G., et al. (2003). Avoiding pitfalls of using species distribution models in conservation planning. Conservation Biology, 17, 1591-1600. 
Luna-Vega, I., Espinosa, D. y Contreras-Medina, R. (2016). Biodiversidad de la Sierra Madre del Sur una síntesis preliminar. México, D.F.: Facultad de Ciencias, UNAM.

Margules, C. R. y Sarkar, S. (2009). Planeación sistemática de la conservación. México, D.F.: Universidad Nacional Autónoma de México, Comisión Nacional de Áreas Naturales Protegidas y Comisión Nacional para el Conocimiento y Uso de la Biodiversidad.

Meléndez-Herrada, A., Chimal, A., Figueroa, A., Vázquez, E. y García, F. M. (2015). Biodiversidad de Barra de Potosí, Guerrero, México: hacia una interacción entre conservación y turismo Número 123, Serie Académicos. México, D.F.: Universidad Autónoma Metropolitana.

Méndez-Larios, I., Villaseñor, J. L., Lira, R., Morrone, J. J., Dávila, P. y Ortiz, E. (2005). Toward the identification of a core zone in the Tehuacán-Cuicatlán Biosphere Reserve, Mexico, based on parsimony analysis of endemicity of flowering plant species. Interciencia, 30, 267-274.

Monteagudo, D. y Luis, M. A. (2013). Patrones de riqueza altitudinal de Papilionidae, Pieridae y Nymphalidae (Lepidoptera: Rhopalocera) en áreas montañosas de México. Revista de Biología Tropical, 61, 1509-1520.

Monterrubio-Rico, T. C., Charre-Medellín, J. F., Pacheco-Figueroa, C., ArriagaWeiss, S., Valdez-Leal, J. D., Cancino-Murillo, R., et al. (2016). Distribución potencial y contemporánea de la familia Psittacidae en México. Revista Mexicana de Biodiversidad, 87, 1103-1117.

Monterrubio-Rico, T. C., Labra-Hernández, M. Á. D., Ortega-Rodríguez, J. M., Cancino-Murillo, R. y Villaseñor-Gómez, J. F. (2011). Distribución actual y potencial de la guacamaya verde en Michoacán, México. Revista Mexicana de Biodiversidad, 82, 1311-1319.

Moreno, C. E. y Halffter, G. (2000). Assessing the completeness of bat biodiversity inventories using species accumulation curves. Journal of Applied Ecology, 37, 149-158.

Morrone, J. J. (1994). On the identification of areas of endemism. Systematic Biology, 43, 438-441.

Morrone, J. J. (2003). El lenguaje de la cladística. México, D.F.: Las Prensas de Ciencias, Facultad de Ciencias, UNAM.

Morrone, J. J. y Crisci, J. V. (1995). Historical biogeography: introduction to methods. Annual Review of Ecology and Systematics, 26, 373-401.

Navarro, A. G. (1992). Altitudinal distribution of birds in the Sierra Madre del Sur, Guerrero, Mexico. Condor, 94, 29-39.

Navarro, A. G. (1998). Distribución geográfica y ecológica de la avifauna del estado de Guerrero, México (Tesis doctoral). México, D.F.: Facultad de Ciencias, Universidad Nacional Autónoma de México.

Navarro, J. C., Liria, J., Piñango, H. y Barrera, R. (2007). Biogeographic area relationships in Venezuela: a parsimony analysis of Culicidae, Phytotelmata distribution in national parks. Zootaxa, 1547, 1-19.

Navarro, A. G. y Peterson, A. T. (1999). Extensión del área de distribución de aves en el oeste de Guerrero, México. Anales del Instituto de Biología, Universidad Nacional Autónoma de México. Serie Zoología, 70, 41-50.

Navarro, A. G., Peterson, A. T. y Gordillo-Martínez, A. (2003). Museums working together: the atlas of the birds of Mexico. Bulletin of the British Ornithologists' Club, 123A, 207-225.

Navarro-Sigüenza, A. G., Blancas-Calva, E., Almazán-Núñez, R. C., GarcíaTrejo, E. A., Hernández-Baños, B. E. y Peterson, A. T. (2016). Diversidad y endemismo de las aves de la Sierra Madre del Sur. En I. Luna, D. Espinosa, y R. Contreras-Medina (Eds.), Biodiversidad de la Sierra Madre del Sur, una síntesis preliminar (pp. 381-411). México, D.F.: Facultad de Ciencias, UNAM.

Navarro-Sigüenza, A. G., Lira-Noriega, A., Arizmendi, M. C., Berlanga, H., Koleff, P., García-Moreno, J. et al. (2011). Áreas de conservación para las aves de México: integrando criterios de priorización. En P. Koleff y T. Urquiza-Haas (Coords.), Conabio-Conanp (coords.), Planeación para la conservación de la biodiversidad terrestre en México: retos en un país megadiverso (pp. 108-129). México, D.F.: Conabio-Conanp.

Navarro-Sigüenza, A. G. y Peterson, A. T. (2004). An alternative species taxonomy of the birds of Mexico. Biota Neotropica, 4, 1-32.

Navarro-Sigüenza, A. G., Rebón-Gallardo, M. F., Gordillo-Martínez, A., Peterson, T. A., Berlanga-García, H. y Sánchez-González, L. A. (2014). Biodiversidad de aves en México. Revista Mexicana de Biodiversidad, 85, 476-495.
Nihei, S. S. (2006). Misconceptions about parsimony analysis of endemicity. Journal of Biogeography, 33, 2099-2106.

Nixon, K. C. (1999). The parsimony ratchet, a new method for rapid parsimony analysis. Cladistics, 15, 407-414.

Ochoa-Ochoa, L. M. y Flores-Villela, O. A. (2002). Áreas de diversidad y endemismo de la herpetofauna mexicana. México, D.F.: UNAM-Conabio.

Ortega-Huerta, M. A. y Peterson, A. T. (2004). Modelling spatial patterns of biodiversity for conservation prioritization in north-eastern Mexico. Diversity and Distributions, 10, 39-54.

Peralta-Gómez, S., Diego-Pérez, N. y Gual-Díaz, M. (2000). Listados florísticos de México XIX. La Costa Grande de Guerrero. México, D.F.: Universidad Nacional Autónoma de México.

Peterson, A. T. (2001). Predicting species' geographic distributions based on ecological niche modeling. The Condor, 103, 599-605.

Peterson, A. T., Egbert, S. L., Sánchez-Cordero, V. y Price, K. P. (2000). Geographic analysis of conservation priority: endemic birds and mammals in Veracruz, Mexico. Biological Conservation, 93, 85-94.

Peterson, A. T. y Navarro-Sigüenza, A. G. (2000). Western Mexico: a significant centre of avian endemism and challenge for conservation. Cotinga, 14, $42-46$.

Peterson, A. T. y Navarro-Sigüenza, A. G. (2016). Bird conservation and biodiversity research in Mexico: status and priorities. Journal of Field Ornihology, 87, 121-132.

Peterson, A. T., Navarro-Sigüenza, A. G. y Benítez-Díaz, H. (1998). The need for continued scientific collecting; a geographic analysis of Mexican bird specimens. Ibis, 140, 288-294.

Peterson, A. T., Papeş, M. y Eaton, M. (2007). Transferability and model evaluation in ecological niche modeling: a comparison of GARP and Maxent. Ecography, 30, 550-560.

Peterson, A. T., Rice, N. H. y Navarro-Sigüenza, A. G. (2004). Geographic variation in the rosy thrush-tanager (Rhodinocichla rosea) complex of Mesoamerica (Aves: Passeriformes). Biota Neotropica, 4, 1-7.

Peterson, A. T., Sánchez-Cordero, V., Martínez-Meyer, E. y Navarro-Sigüenza, A. G. (2006). Tracking population extirpations via melding ecological niche modeling with land-cover information. Ecological Modelling, 195, 229-236.

Ponce-Ulloa, H. E. y Llorente-Bousquets, J. (1993). Distribución de los Siphonaptera (Arthropoda, Insecta) en la Sierra de Atoyac de Álvarez Guerrero, México Publicaciones especiales 11. México, D.F.: Instituto de Biología, UNAM.

Pressey, R. L., Johnson, I. R. y Wilson, P. D. (1994). Shades of irreplaceability: towards a measure of the contribution of sites to a reservation goal. Biodiversity and Conservation, 3, 242-262.

Ramírez-Bastida, P., Navarro-Sigüenza, A. G. y Peterson, A. T. (2008). Aquatic bird distributions in Mexico: designing conservation approaches quantitatively. Biodiversity and Conservation, 17, 2525-2558.

Rodrigues, A. S. y Brooks, T. M. (2007). Shortcuts for biodiversity conservation planning: the effectiveness of surrogates. Annual Review of Ecology, Evolution, and Systematics, 38, 713-737.

Rodrigues, A. S., Gregory, R. D. y Gaston, K. J. (2000). Robustness of reserve selection procedures under temporal species turnover. Proceedings of the Royal Society of London B: Biological Sciences, 267, 49-55.

Rojas-Soto, O. R., Alcántara-Ayala, O. y Navarro-Sigüenza, A. G. (2003). Regionalization of the avifauna of the Baja California Peninsula, Mexico: a parsimony analysis of endemicity and distributional modelling approach. Journal of Biogeography, 30, 449-461.

Rojas-Soto, O. R., Navarro-Sigüenza, A. G. y Espinosa-de los Monteros, A. (2009). Systematics and bird conservation policies: the importance of species limits. Bird Conservation International, 20, 176-185.

Rovito, S. M., Arroyo, M. T. K. y Pliscoff, P. (2004). Distributional modeling and parsimony analysis of endemicity of Senecio in the Mediterraneantype climate area of central Chile. Journal of Biogeography, 21, $1623-1636$.

Sánchez-Cordero, V., Peterson, A. T. y Escalante-Pliego, P. (2001). Modelado de la distribución de especies y la conservación de la diversidad biológica. En H. Hernández, A. García-Aldrete, F. Álvarez, y M. Ulloa (Eds.), Enfoques contemporáneos en el estudio de la diversidad biológica (pp. 359-379). México, D.F.: Instituto de Biología, UNAM y Fondo de Cultura Económica. 
Sánchez-González, L. A., Morrone, J. J. y Navarro-Sigüenza, A. G. (2008). Distributional patterns of the Neotropical humid montane forest avifaunas. Biological Journal of the Linnean Society, 94, 175-194.

Sarkar, S. y Margules, C. R. (2002). Operationalizing biodiversity for conservation planning. Journal of Biosciences, 27, 299-308.

Semarnat (Secretaría de Medio Ambiente Recursos Naturales) (2009). El medio ambiente en México 2009: en resumen. México, D.F.: Secretaría de Medio Ambiente y Recursos Naturales.

Semarnat (Secretaría del Medio Ambiente y Recursos Naturales) (2010). Norma Oficial Mexicana NOM-059-SEMARNAT-2010. Protección ambientalEspecies nativas de México de flora y fauna silvestres-Categorías de riesgo y especificaciones para su inclusión, exclusión o cambio-Lista de especies en riesgo. México, D.F.: Diario Oficial de la Federación. 30 de diciembre de 2010, segunda sección.

Semarnat (Secretaría de Medio Ambiente Recursos Naturales). (2014). Inventario estatal forestal y de suelos-Guerrero. México, D.F: Secretaría del Medio Ambiente y Recursos Naturales y Comisión Nacional Forestal.

Sierra-Morales, P., Almazán-Núñez, R. C., Beltrán-Sánchez, E., Ríos-Muñoz, C. A. y Arizmendi, M. C. (2016). Distribución geográfica y hábitat de la familia Trochilidae (Aves) en el estado de Guerrero, México. Revista de Biología Tropical, 64, 363-376.

Soberón, J., Osorio-Olvera, L. y Peterson, T. (2017). Diferencias conceptuales entre modelación de nichos y modelación de áreas de distribución. Revista Mexicana de Biodiversidad, 88, 437-441.

Stockwell, D. R. B. y Noble, I. R. (1992). Induction of sets of rules from animal distribution data: a robust and informative method of data analysis. Mathematics and Computers in Simulation, 33, 385-390.

Stockwell, D. y Peters, D. (1999). The GARP modelling system: problems and solutions to automated spatial prediction. International Journal of Geographical Information Science, 13, 143-158.
Suárez-Mota, M. E., Villaseñor, J. L. y López-Mata, L. (2015). La región del Bajío, México y la conservación de su diversidad florística. Revista Mexicana de Biodiversidad, 86, 799-808.

Tsoar, A., Allouche, O., Steinitz, O., Rotem, D. y Kadmon, R. (2007). A comparative evaluation of presence-only methods for modelling species distribution. Diversity and Distributions, 13, 397-405.

Urbina-Cardona, J. N. y Flores-Villela, O. (2010). Ecological-niche modeling and prioritization of conservation-area networks for Mexican herpetofauna. Conservation Biology, 24, 1031-1041.

Urquiza-Haas, T. y Alarcón, A. (2011). Diversidad y complementariedad de especies de vertebrados terrestres en las ecorregiones de nivel IV. En P. Koleff y T. Urquiza-Haas (Eds.), Planeación para la conservación de la biodiversidad terrestre en México: retos en un país megadiverso (pp. 50-51). México, D.F: Conabio-Conanp.

Vane-Wright, R. I. (1996). Systematics and the conservation of biological diversity. Annals of the Missouri Botanical Garden, 83, 47-57.

Vane-Wright, R. I., Humphries, C. J. y Williams, P. H. (1991). What to protect? Systematics and the agony of choice. Biological Conservation, 55, 235-254.

Venegas-Barrera, C. S. y Manjarrez, J. (2011). Patrones espaciales de la riqueza específica de las culebras Thamnophis en México. Revista Mexicana de Biodiversidad, 82, 179-191.

Villaseñor, J. L. y Gual-Díaz, M. (2014). El bosque mesófilo de montaña en México y sus plantas con flores. En M. Gual-Díaz y A. Rendón-Correa (Eds.), Bosques mesófilos de montaña de México: diversidad, ecología y manejo (pp. 221-235). México, D.F.: Conabio.

Wiens, J. A. (1989). . The ecology of bird communities, foundations and patterns (1) Cambridge: Cambridge University Press.

Zamora-Manzur, C., Parra, L. E. y Jaque, E. (2011). Patrones de distribución de los geométridos de la región del Biobío, Chile: una aproximación para su conservación. Revista Chilena de Historia Natural, 84, 465-480. 